\title{
Investigating iodine-induced stress corrosion cracking of zirconium alloys using quantitative fractography
}

DOI:

10.1016/j.jnucmat.2020.152272

\section{Document Version}

Accepted author manuscript

Link to publication record in Manchester Research Explorer

\section{Citation for published version (APA):}

Gillen, C., Garner, A., Anghel, C., \& Frankel, P. (2020). Investigating iodine-induced stress corrosion cracking of zirconium alloys using quantitative fractography. Journal of Nuclear Materials, 539, 152272. [152272].

https://doi.org/10.1016/j.jnucmat.2020.152272

\section{Published in:}

Journal of Nuclear Materials

\section{Citing this paper}

Please note that where the full-text provided on Manchester Research Explorer is the Author Accepted Manuscript or Proof version this may differ from the final Published version. If citing, it is advised that you check and use the publisher's definitive version.

\section{General rights}

Copyright and moral rights for the publications made accessible in the Research Explorer are retained by the authors and/or other copyright owners and it is a condition of accessing publications that users recognise and abide by the legal requirements associated with these rights.

\section{Takedown policy}

If you believe that this document breaches copyright please refer to the University of Manchester's Takedown Procedures [http://man.ac.uk/04Y6Bo] or contact uml.scholarlycommunications@manchester.ac.uk providing relevant details, so we can investigate your claim.

\section{OPEN ACCESS}




\title{
Manuscript Details
}

\section{Manuscript number}

Title
JNM_2019_1505_R2

Investigating lodine-Induced Stress Corrosion Cracking of Zirconium Alloys Using Quantitative Fractography

Full Length Article

\section{Article type}

Abstract

Pellet Cladding Interaction $(\mathrm{PCl})$ is a fuel failure phenomenon that severely limits power manoeuvrability of light water nuclear reactors with lodine-Stress Corrosion Cracking (I-SCC) the most commonly hypothesised cause. To increase mechanistic understanding, I-SCC tests have been performed by compressing zirconium alloy c-rings at fixed load while submerged in iodised ethanol. Samples left until failure produced fracture surfaces subsequently analysed using quantitative SEM fractography, and samples with cracks arrested part way produced incipient cracks assessed by optical imaging. The influence of microstructure, stress and iodine concentration on I-SCC performances has been assessed by comparison between cold-worked and recrystallised material. Increasing the stress resulted in faster time to failure and produced smaller regions of intergranular cracking; explained using the stress intensity factor and its control over the prevalent mechanism, further evidenced by tests that removed either iodine or stress part way through cracking. Increasing iodine concentrations resulted in faster times to failure and more intergranular cracking indicating the importance of an incubation period and a possible crack blunting effect. Cracks in cold-worked material progress at a non-radial angle, whereas cracks within recrystallised and stress-relieved material cracks progress radially. Analysis suggests that the mechanisms that lead to this difference may be related to grain morphology rather than residual stress as is typically suggested in literature, with fractography and incipient cracks used as evidence.

\author{
Manuscript category \\ Nuclear fuels and materials \\ Corresponding Author \\ Conor Gillen \\ Corresponding Author's \\ University of Manchester \\ Institution \\ Order of Authors \\ Conor Gillen, Alistair Garner, Clara Anghel, Philipp Frankel \\ Suggested reviewers \\ Matthew Topping, Sean Hanlon, Markus Piro
}

\section{Submission Files Included in this PDF}

File Name [File Type]

cover letter.docx [Cover Letter]

Response to reviewers.docx [Response to Reviewers]

Parametric Study in ZIRLO post review draft 3 tracked changes.docx [Revised Manuscript with Changes Marked]

Parametric Study in ZIRLO post review draft 3.docx [Manuscript File]

declaration-of-competing-interests.docx [Conflict of Interest]

Author Statement.docx [Author Statement]

To view all the submission files, including those not included in the PDF, click on the manuscript title on your EVISE Homepage, then click 'Download zip file'.

\section{Research Data Related to this Submission}

There are no linked research data sets for this submission. The following reason is given:

Data will be made available on request 
Conor Gillen

Materials Performance Centre

School of Materials

The University of Manchester

Manchester

M13 9PL

UK

Conor.Gillen@manchester.ac.uk

\section{G. S. Was}

Editor-in-Chief

Journal of Nuclear Materials

January 212020

The enclosed paper, entitled 'Investigating lodine-Induced Stress Corrosion Cracking of Zirconium Alloys Using Quantitative Fractography' is being submitted to the Journal of Nuclear Materials. No previous or concurrent submissions have been made and all authors have authorized submission to the journal.

The authors believe this is a novel manuscript that uses quantitative fractography to investigate iodine-stress corrosion cracking (I-SCC) of zirconium alloys while varying stress and iodine quantities. The amounts of intergranular, transgranular and ductile tearing were recorded as the parameters which control I-SCC were changed. Furthermore, variations upon standard procedures of producing I-SCC were performed, with the results having important implications about the mechanism of I-SCC of zirconium alloys.

We believe the manuscript is appropriate for publication in the Journal of Nuclear Materials as the study of Pellet Cladding Interaction, the cause of I-SCC, has a long history of publications within the journal; and the research is directly focused on materials with nuclear fission applications.

Sincerely,

Conor Gillen 
The authors have addressed the comments individually below (bold) and have edited a revised version of the manuscript accordingly, with changes highlighted.

Thank you to both reviewers for the comments.

Conor Gillen

-Reviewer 2

This paper presents high-quality fractography results on I-SCC cracks in ZIRLO material after different heat treatments. Understanding how material and environmental changes affect I-SCC mechanisms and time to failure is of value to validate mechanistic or empirical PCI models. Publication is recommended.

The authors have effectively addressed the previous reviewer comments. Only a few minor follow-up comments remain. Author comment disposition in italics.

The authors have tried to make quantitative comparisons between fracture surfaces but acknowledge there is an inherent subjectivity. Data has been generated from fractographic analysis of 100 regions for each sample, and the results represent the quantitative comparison of all these measurements.

Recommend that this description (or similar), including 'inherent subjectivity', be included in the text.

The following sentence has been added to Section 2.4: "This method generates quantitative data for comparison between samples, although there is an inherent subjectivity in each of the individual observations."

In reactor pitting is often not observed [18-20]. Not all references are related to irradiated material the passage implies pitting is 'always' observed out of reactor. Suggest re-wording.

One reference has been removed. "Typically" has been added to the passage discussing pitting out of reactor.

Tests were also performed on material that had been heated at $300^{\circ} \mathrm{C}$ in an attempt to reduce the residual stress and so examine its effect. If the authors are unsure how successful the attempt was (no quantitative measurements or calculation from a reference demonstrating the expected change in residual stress), consider whether including these 'heat-treated' results add value to the paper.

The authors believe the results do add some value, as residual stress could be important, although recognise that due to the manner it has been investigated the value is limited.

A previous comment regarding units of iodine concentration was likely misinterpreted, which was in part due to an error made by the reviewer. The units used by the authors are $\mathrm{g} / \mathrm{L}$, grams of iodine per litre of ethanol. Previous studies have used other solvents or cover gases, such as methanol, nitrogen, argon, helium, or an evacuated chamber, where many have reported iodine concentrations in the units 
of $\mathrm{mg} / \mathrm{cm} 3, \mathrm{mg}$ of iodine per cubic centimetre of chamber volume (not including the sample). Since $1 \mathrm{~g} / \mathrm{L}$ $=1 \mathrm{mg} / \mathrm{cm} 3$, the reviewer was previously incorrect that the conversion would require density information. However, the prevalence of $\mathrm{mg} / \mathrm{cm} 3$ (and $\mathrm{mg} / \mathrm{cm} 2$ ) in previous studies and the potential confusion in using litres of ethanol as a volume lead to the same reviewer recommendation to use units of $\mathrm{mg} / \mathrm{cm} 3$, where the conversion is $1: 1$ with the values currently in the paper. The reviewer does not suggest using the volume of the $\mathrm{Zr}$ samples (this appears to be the author interpretation of the previous reviewer comment).

The authors thank the reviewer for the clarification, the predominant units used throughout the paper have been changed from $\mathrm{g} / \mathrm{L}$ to $\mathrm{mg} / \mathrm{cm}^{3}$. 


\section{InVEStigating IOdine-InduCEd Stress CORROSion CRACKing OF Zirconium AlLoYs using Quantitative Fractography}

Conor Gillen ${ }^{1 *}$, Alistair Garner ${ }^{1}$, Clara Anghel ${ }^{2}$ Philipp Frankel ${ }^{1}$

${ }^{1}$ Materials Performance Centre, School of Materials, The University of Manchester, Manchester M13 9PL, UK

${ }^{2}$ Westinghouse Electric Sweden AB, SE-721 63, Västerås, Sweden

*Corresponding Author

\section{Abstract}

Pellet Cladding Interaction (PCI) is a fuel failure phenomenon that limits power manoeuvrability of light water nuclear reactors with Iodine-Stress Corrosion Cracking (I-SCC) the most commonly hypothesised cause. To increase mechanistic understanding, ISCC tests have been performed by compressing zirconium alloy c-rings at fixed load while submerged in iodised ethanol. Samples left until failure produced fracture surfaces subsequently analysed using quantitative Scanning Electron Microscopy (SEM) fractography, and samples with cracks arrested part way produced incipient cracks assessed by optical imaging. The influence of microstructure, stress and iodine concentration on I-SCC performances has been assessed by comparison between cold-worked and recrystallised material.

Increasing the stress resulted in faster time to failure and produced smaller regions of intergranular cracking; explained using the stress intensity factor and its control over the prevalent mechanism, further evidenced by tests that removed either iodine or stress part way through cracking. Increasing iodine concentrations resulted in faster times to failure and more intergranular cracking indicating the importance of an incubation period and a possible crack blunting effect.

Cracks in cold-worked material progress at a non-radial angle, whereas cracks within recrystallised and stress-relieved material cracks progress radially. Analysis suggests that the mechanisms that lead to this difference may be related to grain morphology rather than residual stress as is typically suggested in the literature, with fractography and incipient cracks used as evidence. 


\section{Introduction}

PCI is a fuel failure phenomenon that Limits the power manoeuvrability of Light Water Reactors. Resistance to PCI type of degradation is an increasingly desirable attribute as nuclear power seeks to become a base load power source that can respond to power fluctuations in renewable energy sources.

As power is increased, differential thermal expansion of the fuel and cladding leads to regions of high tensile stress on the inner surface of the cladding. The effect is compounded by the production of fission gases during operation, leading to internal pressure increases and fuel swelling [1-3]. These aggressive fission products can attack the inner surface of the cladding and in combination with the stress can lead to fuel failures at stresses that would otherwise be within safe limits [4]. One of the fission products released during operation that can generate PCI degradation is iodine although the contributing effect of other fission products has not been ruled out $[1,5,6]$.

Mitigation against PCI failures in PWRs is achieved primarily by following strict operating limits on power ramp rates, ensuring that the stress encountered by the fuel cladding while fission products are present remains below an empirically derived threshold $[2,5,7]$. The risk of fuel failure through PCI is increased after Extended Reduced Power Operation (ERPO) and in high burnup fuel assemblies [8]. With the drive towards higher burnup and increased power flexibility in modern reactor designs, a better understanding of the PCI phenomenon is required in order to reduce or remove over-conservative empirically-derived stress thresholds whilst ensuring the integrity of the cladding.

The effect of stress $[7,9]$, metallurgical state [10-13] and iodine concentration $[1,6,14]$ upon I-SCC failure rates have been investigated previously, using mainly Zircaloy-2 or Zircaloy-4 material. However, little work has been done investigating the effect of external variables upon the cracking mechanism [11], a gap this work seeks to address and with the aforementioned increased drive for better PCI performance in Pressurised Water Reactors (PWRs), particularly more understanding is required for $\mathrm{Nb}$ containing alloys such as $\mathrm{ZIRLO}^{\mathrm{TM}}, 1$.

Previous attempts to simulate iodine-induced stress corrosion cracks in the laboratory have been performed using gaseous iodine at reactor temperature, as well as iodised alcohol solutions at room temperature for experimental practicality $[1,13,14]$. Iodised alcohol produces cracking with fractography revealing both intergranular and transgranular mechanisms, including both pseudo-cleavage and fluting as observed in reactor created cracks [15]. In addition, the propensity of cracking in both vapour 
and solution responds in the same way to changes in applied stress and iodine concentration [13-15]. The generally agreed upon crack mechanism in I-SCC failures in both experiments involves a crack initiating step through intergranular (IG) pitting, followed by a region of transgranular (TG) pseudo-cleavage cracking and finally ductile failure. In reactor pitting is often not observed $[16,17]$. A key observation is that fracture surfaces created in gaseous iodine show regions of entirely transgranular cracking, while those cracked in alcoholic solutions exhibit transgranular and intergranular cracking occurring simultaneously [15]. Key differences between using iodine in the two forms are: the sizes of the active species, with steric hinderance limiting the movement of iodised alcoholic compounds when compared with iodine vapour [18], and the large difference in temperature: room temperature versus reactor operating temperatures.

In this study, C-ring specimens prepared from cold-worked, stress-relieved and recrystallised ZIRLO cladding specimens, are loaded in compression at different levels and combinations of stress and iodised-ethanol concentrations in order to induce stress corrosion cracks. The PCI behaviour of this alloy has recently become more important with the increased desirability of power manoeuvrability in PWRs, and the laboratory tests described allow a wide range of parameters to be investigated far more easily than would be possible with irradiated samples. Failed samples are analysed and compared through fractographic analysis using scanning electron microscopy (SEM) in order to determine the effect of testing parameters on active cracking mechanism. By varying the testing parameters and analysing the effect upon the cracking mechanism, improved knowledge about the PCI cracking mechanism itself and how it can be controlled can be obtained [19]. Improved mechanistic understanding of I-SCC with detailed microstructural characterization will provide a better understanding of the PCI phenomenon that can help to improve PCI modelling.

1 - ZIRLO is a trademark of Westinghouse Electric Company LLC, its Affiliates and/or its Subsidiaries in the United States of America and may be registered in other countries throughout the world. All rights reserved. Unauthorized use is strictly prohibited. 


\section{Experimental Methods}

\section{1 Materials}

The material used in this study is the commercial alloy ZIRLO (Table 1), provided by Westinghouse, in cold-worked and recrystallised condition. The material was provided in tube form and both conditions had undergone typical commercial processing routes including forging and pilgering with a final annealing step for the recrystallised alloy. The typical cold-worked and recrystallised microstructures are highlighted in Figure 1. Additionally, some of the cold-worked material was heated under vacuum at $300^{\circ} \mathrm{C}$ for five hours allowing the effect of residual stress to be examined.

Cold worked ZIRLO material features long and thin grains with approximate dimensions of $2 \times 10 \mu \mathrm{m}$, with their major axis parallel with the axial direction of the tube, although with $30^{\circ}$ rotation towards the tangential direction due to the pilgering process. In the radial direction the CW grains are $\sim 2 \mu \mathrm{m}$ thick [20]. Recrystallisation leads to a strengthening of the split basal, a $30^{\circ}$ rotation of the prismatic planes away from the radial direction towards the tangential direction, and the formation of $\sim 2 \mu \mathrm{m}$ equiaxed grains in all directions. In both cases, a split basal texture exists in the radial direction. 
Table 1: Composition in wt.\% of ZIRLO alloy used in this study

\begin{tabular}{ccccccc}
\hline Element & $\mathbf{C r}$ & $\mathbf{F e}$ & $\mathbf{N b}$ & $\mathbf{N i}$ & $\mathbf{S n}$ & $\mathbf{Z r}$ \\
\hline Content (wt.\%) & $<0.01$ & 0.09 & 0.87 & $<0.01$ & 0.92 & bal. \\
\hline \hline
\end{tabular}

(a)
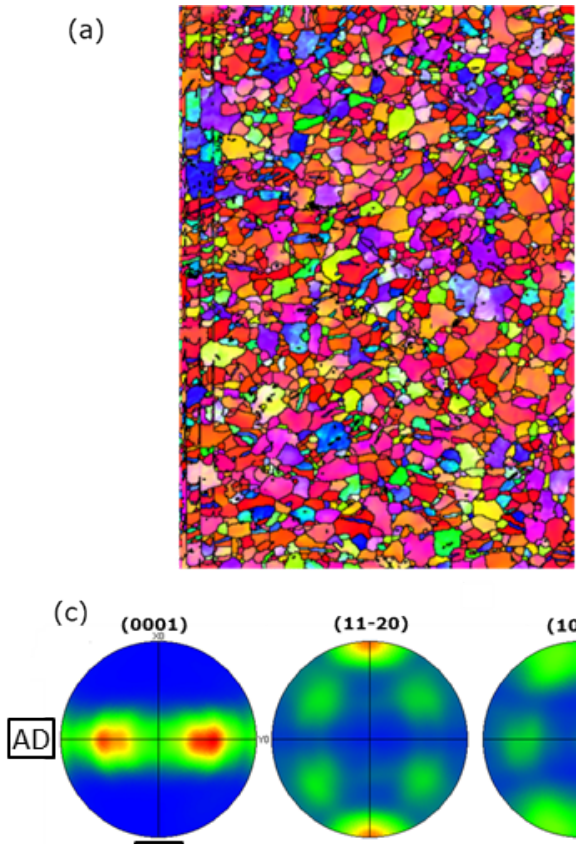

$\mathrm{TD}$

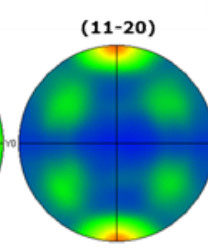

$(11-20)$
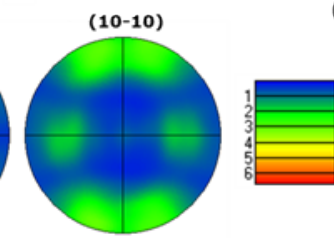

(b)
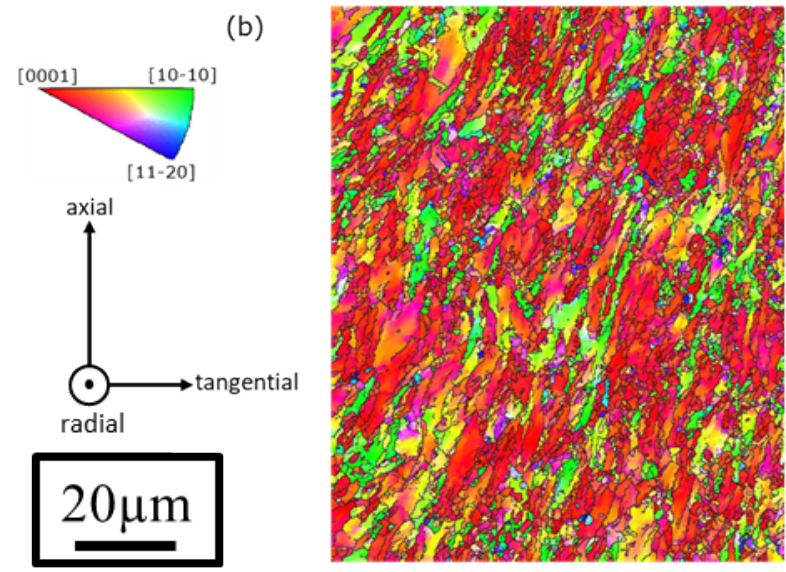

(d)

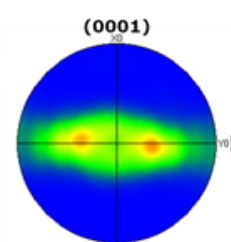

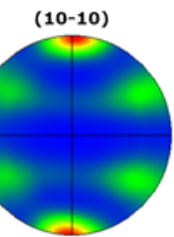

Figure 1 - Electron Backscatter Diffraction (EBSD) orientation maps in IPF-RD (radial tube direction) colouring for (a) Recrystallised and (b) cold-worked ZIRLO cladding tubes. Corresponding pole figures are shown for recrystallised and cold-worked conditions in (c) and (d) respectively.

The tubes were sectioned in order to produce C-ring samples for testing. The C-ring geometry is commonly used in stress corrosion cracking experiments, providing a region of stress concentration without notching so that crack initiation can be investigated [21-25]. The geometry of cladding tubes naturally lends itself to C-ring production and the maximum tensile stress when a displacement is exerted can be calculated using the following formula [21]:

Equation 1

$$
\sigma=\frac{3 E t\left(w-w_{0}\right)}{3 \pi R}
$$

where $E=$ elastic modulus (taken as $90 \mathrm{GPa}[26]$ ), $t=$ wall thickness, $w$ and $w_{0}$ are the slot width of the C-ring in the compressive direction during and before loading respectively, and $R$ is the radius. If the sample is stressed sufficiently as to undergo plastic deformation, this stress is multiplied by a plastic correction factor $f_{p}$, calculated by measuring the amount that the slot width changes when stressed by different displacements according to Sejnoha and Wood [27]: 
Equation 2

$$
f_{p}=\frac{w-\left(w_{u}+w_{R}\right)}{w-w_{0}}
$$

Where $w_{u}=$ the slot width on removing the load and $w_{R}$ the slot width arising from residual stress apparent when first cutting the ring. This factor was calculated for each of the stress levels used in this study. The manufactured samples had a surface area of $59 \mathrm{~cm}^{3}$ and a volume of $0.153 \mathrm{~cm}^{3}$

\subsection{Iodised Ethanol}

The experiments were conducted using $50 \mathrm{~mL}$ of an iodised ethanol solution of varying concentrations, from $0.1 \mathrm{~g}$ of iodine per litre of ethanol up to $20 \mathrm{~g}(\mathrm{I}) / \mathrm{L}(\mathrm{eth})$. This is equivalent to 0.1 to $20 \mathrm{mg}$ of iodine per $\mathrm{cm}^{3}$ of ethanol, units commonly used in previous studies of I-SCC. These values are also roughly equivalent to $\left(\sim 10^{-4}\right.$ to $2 \times 10^{-2}$ grams of iodine + per $\mathrm{cm}^{22}$ of exposed zirconium. $)$ - These concentrations were chosen to cover a similar range of iodine concentrations as Wood et al. [28], ranging from $1 \times 10^{-4}$ to $1 \times$ $10^{-2} \mathrm{~g} / \mathrm{cm}^{2}$. Samples tested only in ethanol, with no iodine presentee, did not exhibit failure. This was done as a controlled reference experimented to demonstrate iodine and not ethanol as the corrosive media.

\subsection{Tensile test conditions}

C-ring samples were tested in compression using an Instron 3344 tensile tester, partially submerged in iodised ethanol within a glass container as shown in Figure 2. Four different strategies were followed to investigate the various effects of iodine and applied stress. Additional tests also investigated the effects of metallurgical condition. All compressions took place at a rate of $2 \mathrm{~mm} / \mathrm{min}$ until the desired displacement (and thus stress) was reached at which point the sample was held under

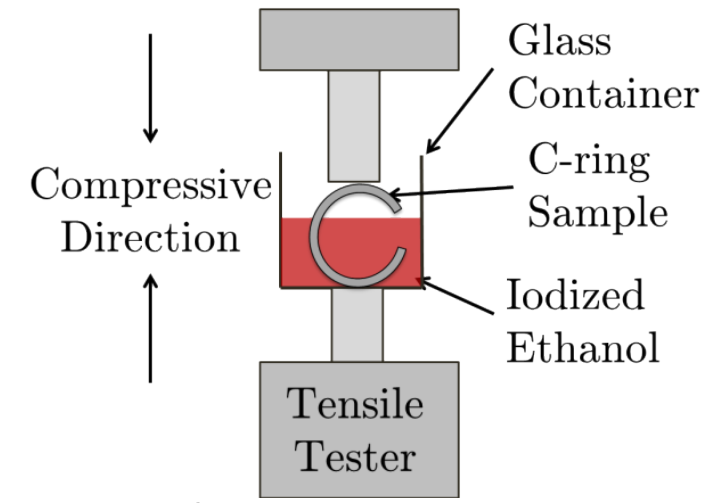

Figure 2 - Experimental setup for C-ring compression tests fixed load to negate plastic relaxation effects. Experiments ran until sample failure.

A displacement vs time graph of a typical test can be seen in Figure 3. Samples are loaded at constant displacement until reaching the desired displacement $(2 \mathrm{~mm})$ at which point they are held at fixed load until cracking begins. The tests were performed at 


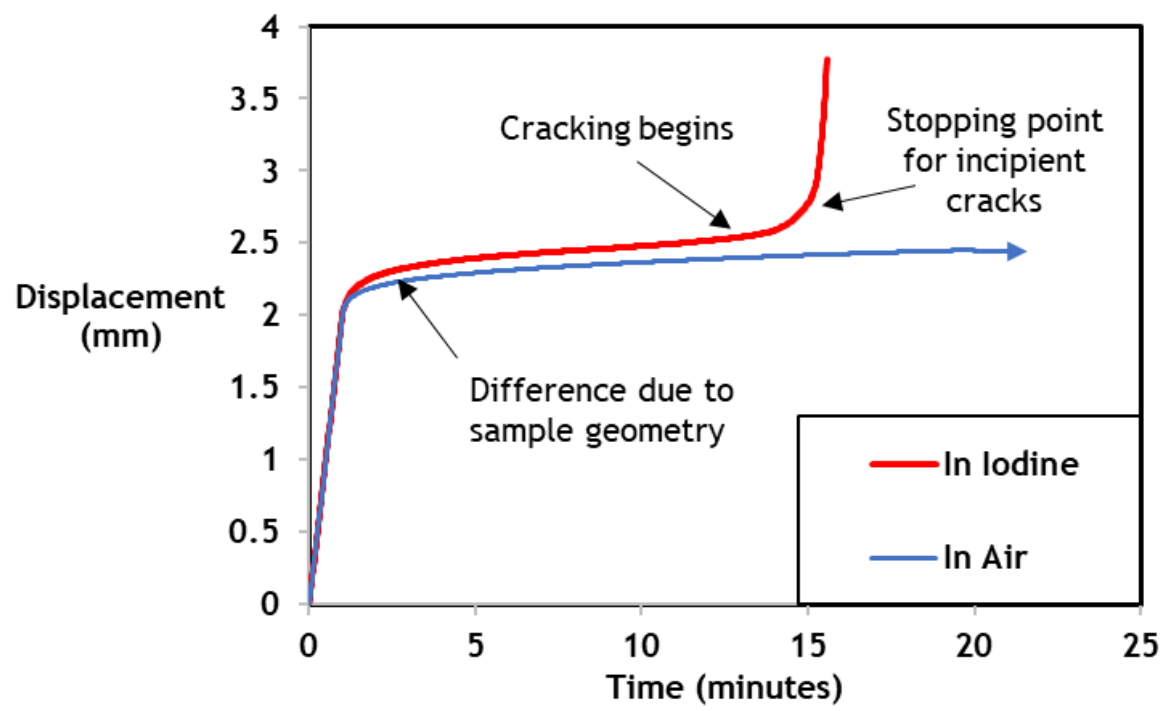

Figure 3 - Typical displacement vs time behaviour for cold-worked C-ring samples compressed to $2 \mathrm{~mm}$ and then held at fixed load in iodine-ethanol solution and in air.

properties differ between the material conditions, the resultant stresses on each material condition were different.

\subsubsection{Varying Macroscopic Stress}

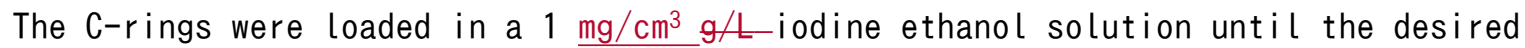
maximum tensile stress ranging from 325 - $750 \mathrm{MPa}$ on the outer C-ring surface was reached (according to Equation 1), before holding at constant load.

Table 2: The four experiments investigating the effect of iodine concentration and annlied stress

\begin{tabular}{|c|c|c|c|c|}
\hline Experiment & $\begin{array}{l}\text { Initial crack } \\
\text { conditions }\end{array}$ & $\begin{array}{c}\text { Initial Crack } \\
\text { propagation stopped at }\end{array}$ & $\begin{array}{c}\text { Second crack } \\
\text { growth } \\
\text { conditions }\end{array}$ & $\begin{array}{l}\text { Second crack } \\
\text { stopped at }\end{array}$ \\
\hline $\begin{array}{l}\text { Varying } \\
\text { Stress }\end{array}$ & $\begin{array}{c}\text { 397-794 MPa, } 1 \\
\mathrm{mg} / \mathrm{cm}^{3} \mathrm{~g} / \mathrm{t}\end{array}$ & Ran until failure & $\mathrm{n} / \mathrm{a}$ & \\
\hline $\begin{array}{l}\text { Varying } \\
\text { Iodine Conc. }\end{array}$ & $\begin{array}{c}750 \mathrm{MPa}, \\
0.1-20 \\
\mathrm{mg} / \mathrm{cm}^{3} \mathrm{~g} / \mathrm{L}\end{array}$ & Ran until failure & $\mathrm{n} / \mathrm{a}$ & \\
\hline $\begin{array}{l}\text { Temporary } \\
\text { removal of } \\
\text { Stress }\end{array}$ & $\begin{array}{c}750 \mathrm{MPa}, \\
1 \mathrm{mg} / \mathrm{cm}^{3} \mathrm{~g} / \mathrm{L}\end{array}$ & $\begin{array}{c}0.5 \mathrm{~mm} \text { change in } \\
\text { displacement }\end{array}$ & $\begin{array}{c}750 \mathrm{MPa}, \\
1 \mathrm{mg} / \mathrm{cm}^{3} \mathrm{~g} / \mathrm{L}\end{array}$ & Ran until failure \\
\hline $\begin{array}{l}\text { Removal of } \\
\text { Iodine }\end{array}$ & $\begin{array}{c}750 \mathrm{MPa}, \\
1 \mathrm{mg} / \mathrm{cm}^{3} \mathrm{~g} / \mathrm{L}\end{array}$ & $\begin{array}{c}0.5 \text { - } 1 \mathrm{~mm} \text { change in } \\
\text { displacement }\end{array}$ & $750 \mathrm{MPa}$, in air & $\begin{array}{l}\text { Ran until failure or } \\
\text { crushed }\end{array}$ \\
\hline
\end{tabular}




\subsubsection{Varying lodine Concentration}

C-rings were compressed in varying concentrations of iodine ethanol solutions from $0.1-20 \mathrm{mg} / \mathrm{cm}^{3} \mathrm{~g} / \mathrm{L}$ at room temperature until a stress of $750 \mathrm{MPa}$ was reached, at which point constant load was maintained until sample failure or 15 hours elapsed (at which point the sample was considered to have not failed).

\subsubsection{Temporary Removal of Stress}

C-rings were compressed as before at fixed load upon reaching $750 \mathrm{MPa}$ all while in $1 \mathrm{mg} / \mathrm{cm}^{3} \mathrm{~g} / \mathrm{L}$ iodine ethanol solution. Cracking was observed to have begun by a change of 0 . $-5 \mathrm{~mm}$ displacement occurring (as can be seen in Figure 3 ) at which point all applied stress was removed and the sample was left submerged in iodine for up to a week. Following this loading was recommenced in solution as before until sample failure.

\subsubsection{Removal of lodine}

Samples were compressed, until a maximum tensile load on the outer C-ring surface reached $750 \mathrm{MPa}$, at which point fixed load was applied, until a change of displacement ranging from $0.5-1 \mathrm{~mm}$ occurred indicative of incipient cracks. Stress was removed, and samples cleaned in ethanol. Samples were then subjected to the same compressive load as previously subjected to, but in air and not iodine solution. Either failure or survival was recorded following the second compression.

\subsubsection{Effect of Microstructure}

ZIRL0 C-ring samples of either cold-worked, stress-relieved or recrystallised material conditions were compressed to a range of stresses and iodine concentrations until sample failure.

\section{4 Microscopy}

Fractography was performed using a Zeiss EVO 60 SEM at $10 \mathrm{keV}$. Images were taken at 100 evenly spaced locations covering the fracture surface with the dominant fracture mechanism at each recorded, resulting in fracture surface maps of each sample. The proportions of the different fracture mechanisms could then be compared as applied stress and iodine concentrations are varied. This method generates quantitative data for comparison between samples, although there is an inherent subjectivity in each of the individual observations.

For comparison of incipient cracks, some tests were interrupted prematurely, and samples for cross sectional examination were prepared by grinding down non-failed 
samples from the axial direction of the cladding tube, followed by mechanical grinding and polishing, allowing optical images of the crack paths to be taken using a Zeiss Axio Scope. Areas of interest were selected as described in previously published work [29].

\section{Results}

\section{1 Incipient Cracks}

Typical arrested incipient cracks are shown in Figure 4, for both recrystallised and cold-worked material conditions. Cracking in recrystallised material progress with some directional changes on a scale similar to the grain size, but an overall radial direction is maintained. Coldworked material instead begins radially, before changing direction and progressing towards the tangential direction. Off-shoots progress from the main crack towards the opposite tangential direction.

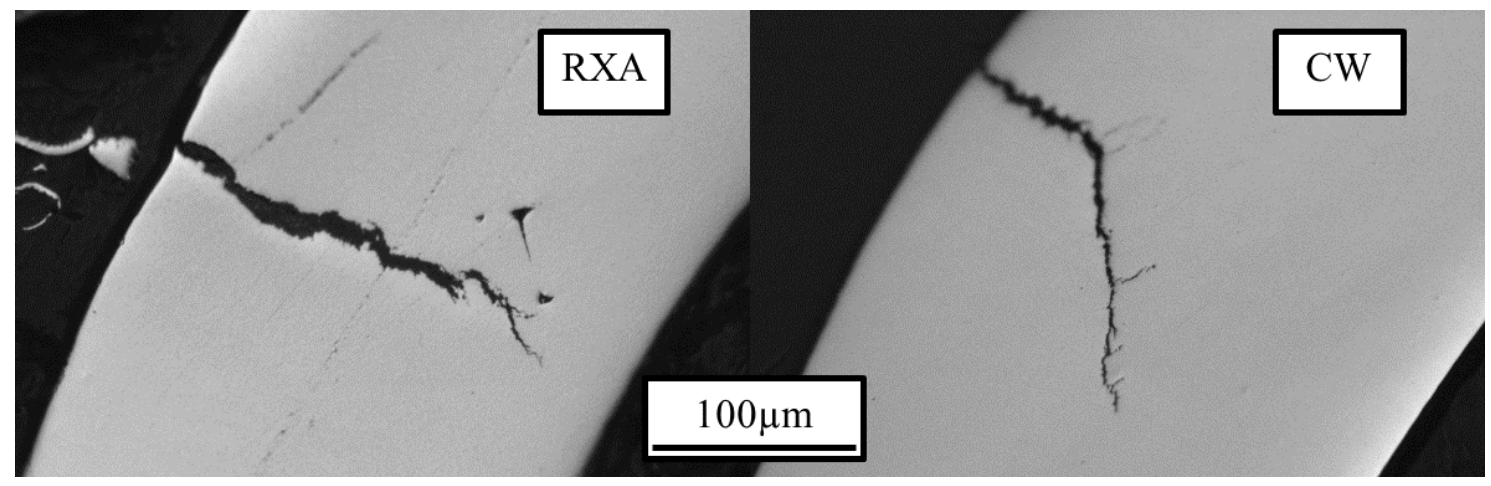

Figure 4 - Incipient cracks in RXA (left) and CW ( $r$ ight) ZIRLO material showing the difference in crack direction. Images are optical micrographs of crack tips polished down from each.

It is possible to use EBSD analysis to provide detailed microstructural analys is of the relationship of the crack tip to the microstructure [29], however that is limited to the very tip of the crack as correlating the crack to adjacent grains is much more difficult once the crack becomes open. In this work the crack path was monitored using fractography to provide information for many more grains.

\section{2 Effect of Microstructure}

The metallurgical state of the material was observed to have a large effect on the resulting crack path, as shown in the optical micrographs in Figure 4. Cracks in recrystallised ZIRLO material generally progress in a radial direction, taking the 
shortest route to the inner surface. In contrast, the cold worked material typically cracks in a direction approximately $50^{\circ}$ away from the radial direction following only a small amount of radial cracking, taking a considerably longer route from the outer to inner surface. In many cases, crack legs were observed to appear at 90 from the main crack path, and in the example in Figure 4, the crack leg was observed to develop into the main crack.

This radial vs non-radial cracking is also apparent in the TG images of RXA and CW material shown in Figure 5 (b) and (e) respectively. Features of similar size and shape to the grains shown in the EBSD map of Figure 1 are indicative of intergranular failure. In other places, RXA material exhibits flat transgranular cracking, with a river pattern typical of cleavage, whereas the CW TG image shows stepped cracking, with cleavage planes interrupted by perpendicular fluting steps. Regions identified as ductile tearing exhibit spherical microvoids, with areas of large ductility between them.

Tests were also performed on material that had been heated at $300^{\circ} \mathrm{C}$ in an attempt to reduce the residual stress and so examine its effect - these showed identical crack morphology to cold-worked material.

\subsection{Effect of Macroscopic Stress}

As the stress was increased, the time to failure for the ZIRLO samples submerged in $1 \mathrm{mg} / \mathrm{cm}^{3} \mathrm{~g} / \mathrm{L}$ iodised ethanol was observed to increase. Figure 6 compares results from the current investigation to results from the literature $[14,26,29]$. 


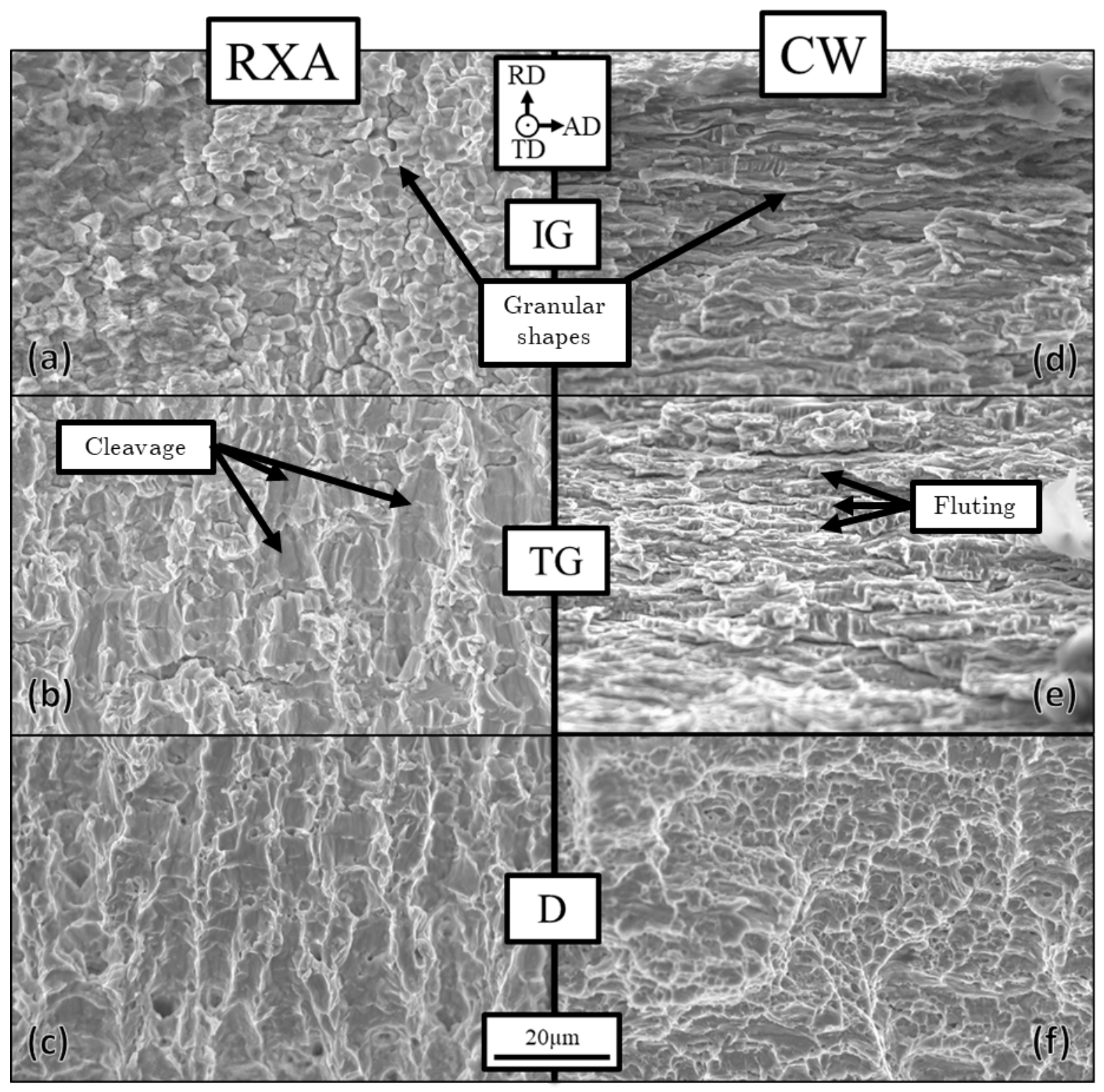

Figure 5 - Fracture surfaces from recrystallized (RX) $(a, b, c)$ and cold worked (CW) (d, e, f) ZIRLO Crings subjected to iodine stress corrosion cracking. Three areas of each are shown, showing the three typically observed regions dominated by the different fracture mechanisms; intergranular cracking (IG) $(a, d)$, transgranular cracking (TG) by pseudo-cleavage and fluting $(b, e)$ and ductile failure (D) (c, f). All images shown have the same magnification and orientation as specified. 


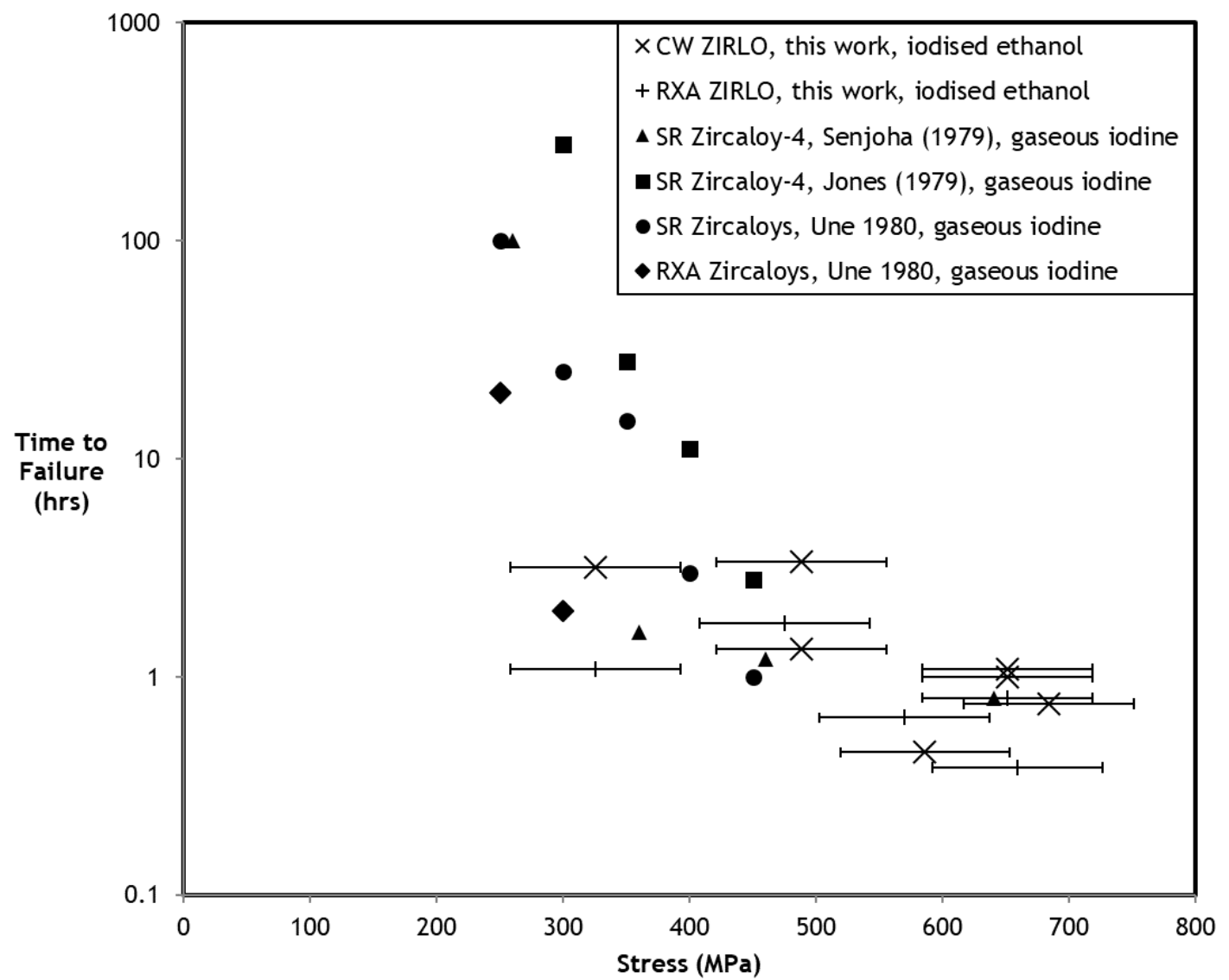

Figure 6 - Variation of time to failure versus changing stress for this work and selected others $[12,27,30]$

Corresponding changes in the proportions of IG, TG by pseudo-cleavage, and ductile cracking is evident on the fracture surface of ZIRLO samples submerged in $1 \mathrm{mg} / \mathrm{cm}^{3} \mathrm{~g} / \mathrm{L}$ iodised ethanol as shown in Figure 7, with increasing stress producing less intergranular cracking and more ductile failure, with the amount of transgranular cracking remaining fairly constant.

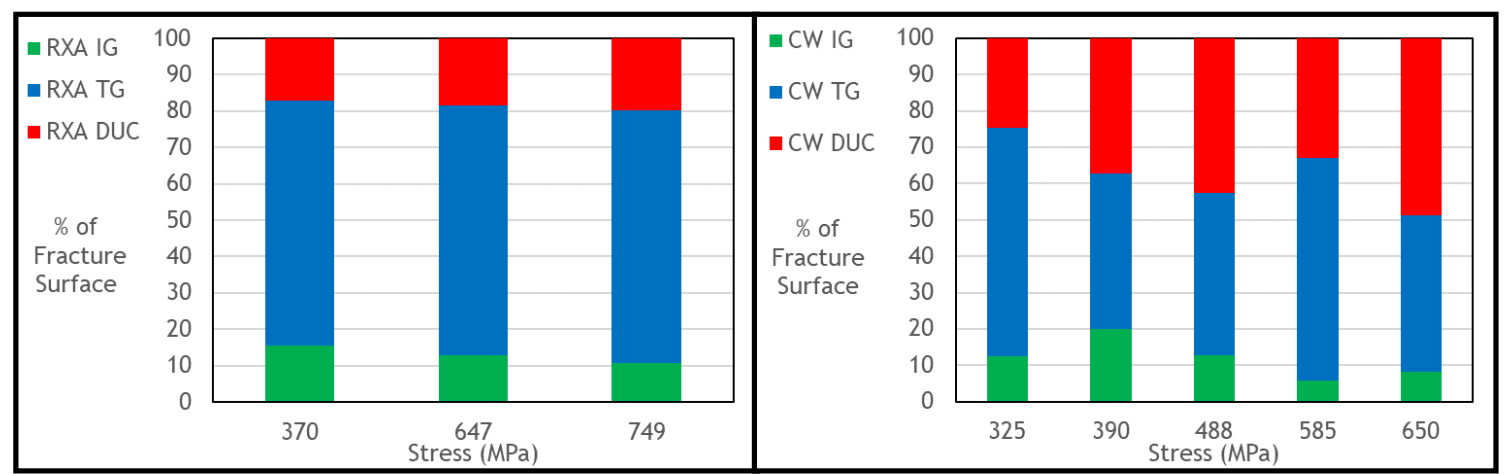

Figure 7 - The effects of applied stress and metallurgical state on the relative proportion of the fracture mechanisms observed by fractography for ZIRLO samples submerged in $1 \mathrm{mg} / \mathrm{cm}^{3} \mathrm{~g} / \mathrm{L}$ iodised ethanol at RT. 


\subsection{Effect of Iodine Concentration}

The effects of changing iodine concentration on the time to failure for C-rings compressed at a constant stress of $750 \mathrm{MPa}$ is shown in Figure 8 . As the iodine concentration was decreased, time to failures were observed to increase up until a concentration of approximately $1 \mathrm{mg} / \mathrm{cm}^{3} \mathrm{~g} / \mathrm{L}$. After this point, increases in concentration do not seem to affect times to failure. This trend is in agreement with previous results $[28,30]$. The value at which saturation begins $\left(1 \underline{\mathrm{mg} / \mathrm{cm}^{3}} \mathrm{~g} / \mathrm{t}\right)$ is equivalent to $\sim 2 \times 10^{-3}$ $\mathrm{g}(\mathrm{I}) \mathrm{cm}^{-2}$ of zirconium cladding, of similar value to $6 \times 10^{-3} \mathrm{~g} / \mathrm{cm}^{2}$ observed by Wood [28]. The clear scatter in the data obfuscates any small difference that may exist in saturation concentration between the two material conditions, however they appear to be of the same magnitude.

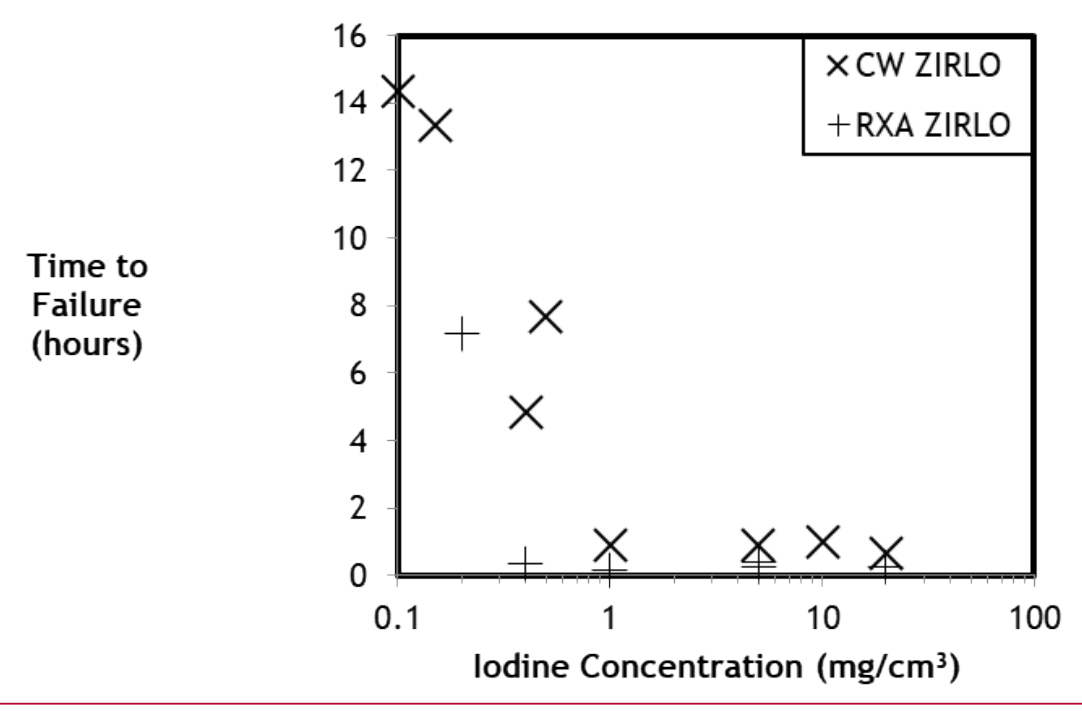

Figure 8 - The effect of changing iodine concentration on time to failure of cold-worked and recrystallised ZIRLO C-rings compressed and held at fixed $750 \mathrm{MPa}$ load until failure while submerged in iodised ethanol solution at RT.

The proportions of the resulting fracture surfaces dominated by each fracture mechanism are plotted in Figure 9. As the iodine concentration is increased, the amount of intergranular and ductile cracking is observed to increase, while the amount of transgranular cracking by pseudo-cleavage decreases. 


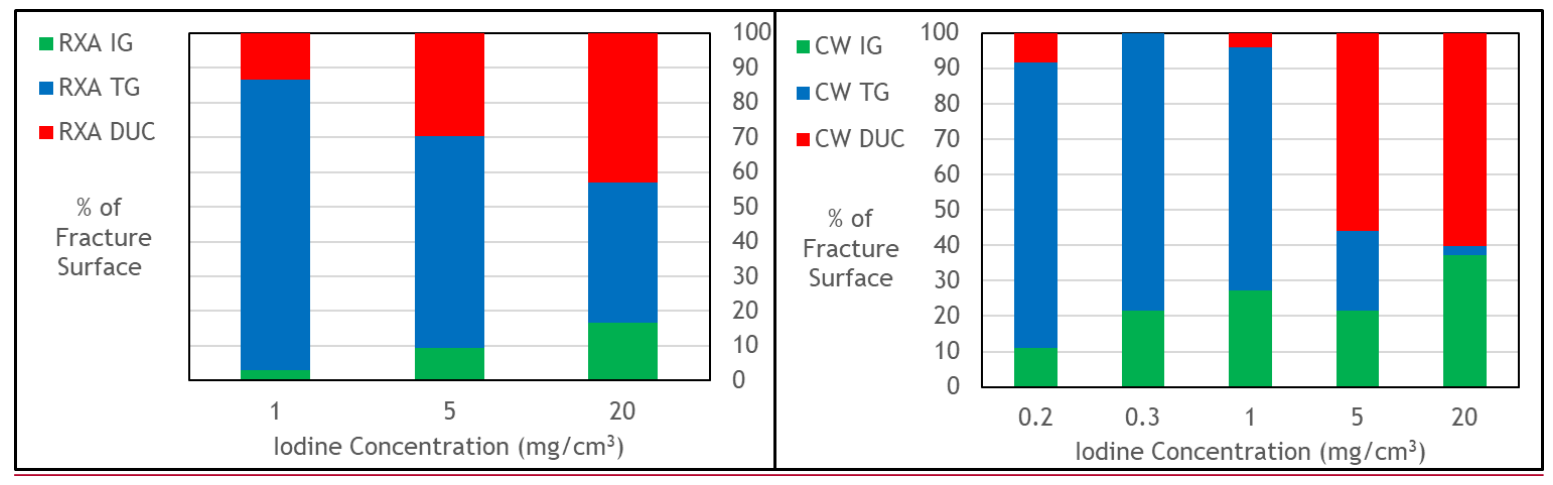

Figure 9 - The effects of changing iodine concentration and metallurgical state upon the distribution of different fracture mechanisms observed by fractography.

It should also be noted that for the exposure of ZIRLO samples to iodine concentrations less than $0.1 \mathrm{mg} / \mathrm{cm}^{3} \mathrm{~g} / \mathrm{t}$, cracking was not observed to occur within 15 hours, as for the ZIRL0 samples that were not exposed to iodine at all. Fracture surfaces from RXA samples tested at $<1 \mathrm{mg} / \mathrm{cm}^{3} \mathrm{~g} / \mathrm{L}$ were not fractographically examined.

\section{5 Temporary Removal of Stress}

Three ZIRL0 samples were partially cracked as described in Section 2.3.3, before being left submerged in iodised ethanol solution but under no applied stress for varying amounts of time, up to one week. The effect of corrosion could be observed by examining the maximum load that the samples reached before failure when subjected to the second compression test, as can be seen in Table 3.

Table 3: Effect of time spent submerged in iodised ethanol solution without stress upon failure load.

\begin{tabular}{|c|c|c|}
\hline Sample & \multicolumn{1}{c}{ Time submerged } & $\begin{array}{c}\text { \% Original } \\
\text { load reached }\end{array}$ \\
\hline (a) & 1 Hour & 97 \\
\hline (b) & 1 Day & 95 \\
\hline (c) & 1 Week & 79 \\
\hline
\end{tabular}

Fracture surfaces were then examined and characterised as illustrated in Figure 10. Sample (a), that was left submerged without stress for an hour, the shortest amount of time, shows no intergranular cracking in the middle of the sample. Sample (b), which was submerged for a day shows a small region of intergranular cracking in the middle of the sample, where only TG cracking would typically be expected. Sample (c), which was left submerged for one week, shows extensive intergranular cracking in the middle of the fracture surface. For samples (b) and (c), deep voids/cracks that extend into the material are surrounding the areas of intergranular cracking, which appear to be caused by intergranular corrosion. 

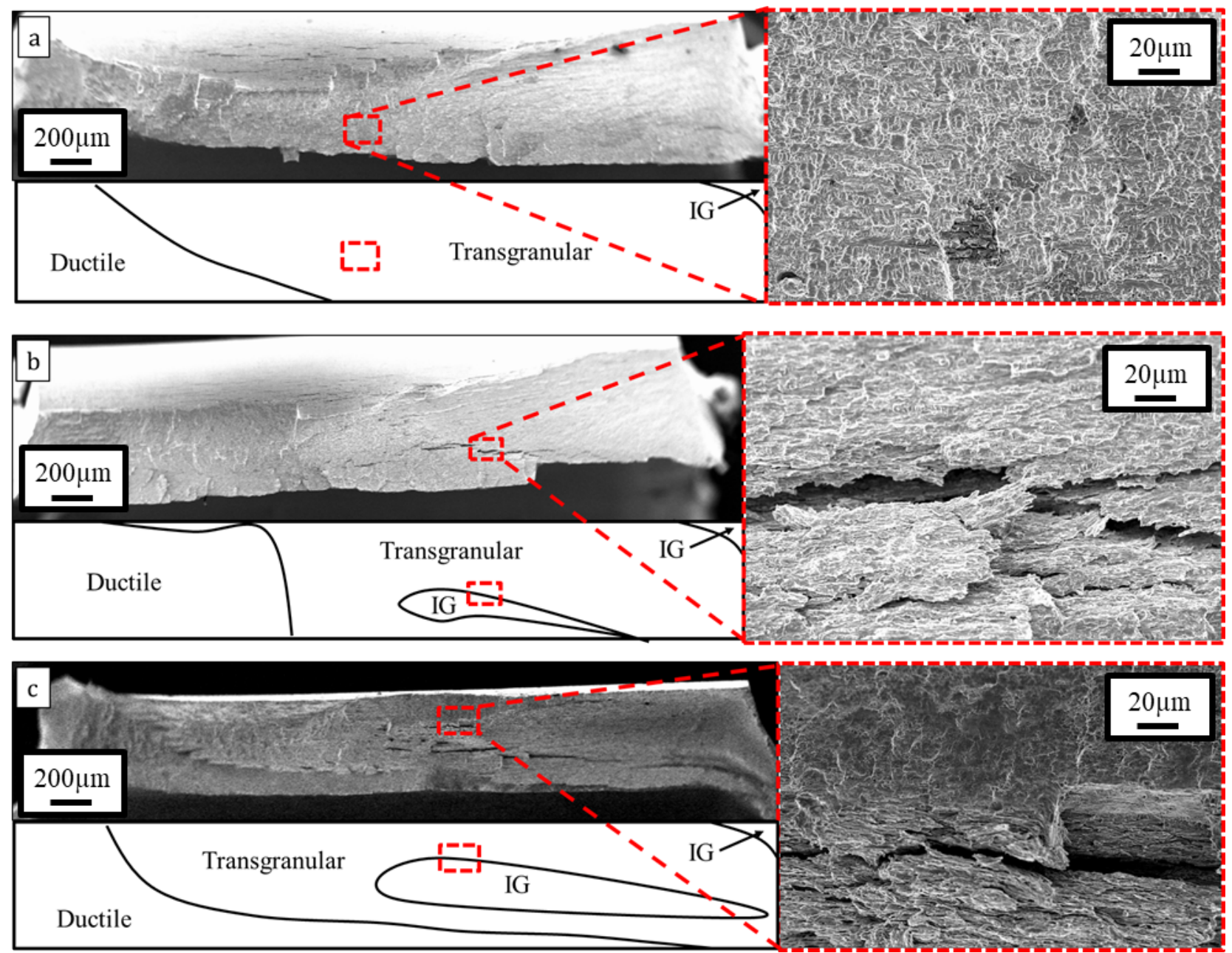

Figure 10 - Fractographic analysis of cold-worked ZIRLO samples partially cracked and then left submerged in iodised ethanol without an applied stress for a. One hour b. One day and c. One week, before being cracked until failure. The fractographic images with higher magnification (right side of the figure) show regions of IG cracking in the middle of the fracture surface or lack thereof.

\section{6 Removal of Iodine}

Three ZIRLO samples were partially cracked to different degrees as described in Section 2.3.4, before being submerged in ethanol and agitated for $\sim 30$ seconds, before being re-subjected to compression without the presence of iodine. When samples were held at fixed load, crack propagation could be seen by changes in crosshead displacement as in Figure 3. The amount of incipient cracking was controlled by removing the stress

Table 4: Effect of initial crack size (measured by change in displacement) upon whether the sample would fail when subsequently subjected to compression without iodine present.

\section{Sample $\quad \Delta$ displacement $(\mathrm{mm}) \quad$ Failure of sample on second compression?}

\begin{tabular}{|c|c|c|}
\hline $\mathbf{1}$ & 0.5 & $\times$ \\
\hline $\mathbf{2}$ & 0.75 & $\times$ \\
\hline $\mathbf{3}$ & 1 & $\checkmark$ \\
\hline
\end{tabular}


and iodine for different changes in this displacement. Whether samples failed or not when subjected to a second compression without iodine was then recorded, as can be seen in Table 4. Only the sample where the initial crack was allowed to grow the most was able to fail when subjected to subsequent compression without iodine present.

\section{Discussion}

Loading of C-rings in iodised ethanol solutions has allowed simulation of I-SCC in the laboratory whilst varying both stress and iodine concentration. The time to failure was observed to decrease as applied stress and iodine concentrations were increased. Fracture surfaces have been quantified by measuring the proportions of different cracking mechanisms present as iodine concentrations have been varied. Increasing stress has reduced the amount of intergranular cracking, whereas increasing iodine concentration has increased the amount of intergranular cracking. The metallurgical state of the material has not been observed to have a significant effect on the proportions of each cracking mechanism observed during fractography, however, incipient cracks in cold-worked and stress relieved ZIRLO have been observed to crack at a different angle than those in recrystallised material. The division of the cladding wall in three cracking patterns (Figure 5) and the time to failure show similarities (Figure 6 and Figure 8) when comparing the CW and RXA materials; this behaviour might be due to the similar split basal texture of all examined microstructures. For the current testing parameters, the time to failure for the CW and RXA materials is quite similar, which indicates that the rate limiting step is not affected by the microstructure of the material.

It is generally accepted within the literature that I-SCC proceeds through intergranular corrosion initially (although cracks beginning with transgranular cracking have been observed [31]), the crack propagates along susceptible grain boundaries (aided by stress) until the stress at the tip of this developing microcrack reaches a critical point. Transgranular cracking then occurs through pseudo-cleavage along basal planes $[12,28]$. This mechanism does not occur in an inert environment - a corrosive media is required and so is not expected to travel as fast as a true cleavage crack would as time for some chemical interaction at the crack tip is required for crack propagation to occur. It is therefore referred to as pseudo-cleavage; and is a SCC process. These cleavage planes can be connected when not aligned by prismatic plane failure, resulting in fluting, observable as the ' $Y$ ' shaped features in Figure 5 . The rest of the cladding wall may finally fail by ductile tearing due to microvoid 
coalescence [32]. This is not an SCC process, since it will occur in the complete absence of any corrosive media if the stress is high enough.

All fracture surfaces examined in this study agree with this sequence of progression of the different cracking mechanisms. Intergranular cracking occurred mostly at the outer C-ring edge where the crack was thought to initiate, and ductile tearing occurred on the inner surface, which would be last to fail. Transgranular cracking by pseudocleavage was mostly observed in the middle portion of the fracture surfaces, between the two other mechanisms, with the exception of the interrupted stress tests where prolonged exposure to iodine without an applied stress resulted in additional intergranular regions.

\section{1 The effect of Stress}

The effect of applied stress upon I-SCC failure rates is perhaps the most investigated relationship in literature and its general effect upon the cracking mechanism is well known and intuitive; increasing stress leads to a decrease in failure times, with the presented results in good agreement with trends reported in the literature $[12,27,30]$. This relationship however was not the primary focus of this work. Macroscopic stress has been previously shown to relate to the increased dominance of TG cracking at higher stresses, a mechanism much faster than IG cracking based upon acoustic emission data and previous comparison of fracture surfaces relative to failure times $[1,33]$. It is this relationship that the current work has sought to investigate. As can be seen from Figure 7, samples under higher stresses exhibit fracture surfaces with less intergranular cracking and more ductile tearing, a transgranular mechanism. For samples undergoing larger macroscopic stress the critical stress intensity factor at which ductile tearing can occur will be reached with a smaller crack length, and thus those samples under large stresses will undergo larger amounts of ductile tearing and will therefore have a decreased time to failure. This is because ductile tearing does not require a chemical contribution and is a fast crack propagation mechanism. It should be noted that the sharpness of the crack tip may also have an important contribution. With less time exposed to iodine, the crack that progresses under a higher macroscopic stress will be sharper, as less crack blunting corrosion can occur at the crack tip.

It is expected that as the stress intensity factor at a crack tip $K$, increases and reaches critical values, different mechanisms of I-SCC are enabled, as shown below:

Table 5: Dominant crack mechanisms for varying stress intensity factors, where $K_{T G}$ is defined as the minimum stress intensity at which transgranular cracking can occur, and $K_{\text {Ductile }}$ the minimum for ductile tearing.

$$
\mathrm{K}: \quad 0<\mathrm{K}_{\mathrm{TG}}<\mathrm{K}_{\text {Ductile }}
$$

\begin{tabular}{l|l|l|l} 
Mechanism: & Intergranular Attack & Pseudo-Cleavage & Ductile
\end{tabular}


Thus the presented results can be explained by considering that the stress intensity factor $K$ can be defined using the macroscopic stress the sample is held at $\sigma$, and some function of the crack length $a$ denoted $F(a)$, such that: $K=\sigma F(a)$. Therefore, if the macroscopic stress is increased, the critical values of $K$ are reached for a smaller crack size and in a shorter amount of time. The resulting fracture surfaces therefore show reduced proportions of IG cracking mechanisms for higher stresses, as the $K_{T G}$ is reached before intergranular attack has had a chance to occur. A number of additional control experiments that temporarily removed stress and completely removed iodine were carried out to separate the effects of different parameters on the resulting fracture surfaces, and lend further evidence for different parts of this explanation.

Firstly, stress was temporarily removed from the sample once an incipient crack had formed. Iodine was then free to attack the zirconium at the crack tip. Only intergranular corrosion occurred, and not a transgranular mechanism, indicating that in the absence of stress the degradation occurs via intergranular attack. This intergranular degraded area was surrounded by areas of transgranular and ductile cracking, presumed to have occurred upon resumption of loading. This fracture surface is atypical for laboratory I-SCC, and only occurred due to the exposure conditions the samples were subjected to. Repeated loading and unloading with the presence of iodine is currently avoided to prevent PCI [8], however may otherwise produce similar fractographic features indicative of the stop-start loading. The presented results are evidence that providing sufficient iodine ( $0.1 \mathrm{mg} / \mathrm{cm}^{3} \mathrm{~g} / \mathrm{L}$ in these experiments) is available intergranular attack can occur with no applied stress, whereas pseudo-cleavage requires enough stress to occur, as suggested in Table 5 .

In a second control test, iodine was removed from testing following the initiation and propagation of incipient cracks to different depths. Subsequent loading without iodine resulted in sample failure for one sample within which the incipient had been allowed to grow the longest. This test provides evidence that ductile tearing can only occur for large incipient cracks and thus large $K$, again as suggested in Table 5; but when high enough $K$ ductile tearing occurs does not require additional iodine to be present.

\section{2 The effect of Iodine concentration}

When examining time to failure (Figure 8), the effect of iodine concentrations may be split into two regimes: 
1. Regime of proportionality in which increasing iodine concentration leads to faster sample failure

2. Saturation regime, during which increases in iodine concentration have no effect upon time to failure.

The two are both observed in the literature $[1,5,28]$. There is a third regime also, not investigated in this work, which occurs at very low iodine concentrations, when there is not enough concentration of corrosive species for I-SCC to initiate by pitting.

The rate limiting step during the regime of proportionality is thought to be the chemical interactions that must take place - zirconium iodides of increasing complexity are thought to form until reaching the volatile $\mathrm{ZrI}_{4}$ molecule, removal of which is believed to be the fundamental mechanism of crack propagation [34]. More local iodine will increase the number of attacked $\mathrm{Zr}$ atoms and increase the likelihood and corrosion rate of the more complex zirconium iodides formation, thus the negative relationship between iodine concentration and time to failure makes sense during the regime of proportionality.

The saturation regime can be explained in the same context, should there be an abundant amount of iodine locally, the amount of zirconium available becomes the restrictive quantity, and further increasing iodine concentrations has no effect, for the investigated stress levels.

Interestingly, the clear change observed between the regime of proportionality and saturation with regard to time to failures is not discernible upon examination of fracture surfaces as shown in Figure 9. Increasing iodine concentrations have resulted in increased levels of intergranular cracking, a difference that persists through the saturation regime, indicating that although the rate limiting step is not affected some other steps may be.

That the amount of intergranular corrosion increases with iodine concentration suggests that in contrast to ductile failure, intergranular corrosion is driven by a chemical process, with stress merely assisting it but not being required. It is intuitive for an increased number of available iodine atoms to cause an increased amount of corrosion through the formation of zirconium iodides. If a protective oxide exists, rupture of that oxide by stress is necessary for crack initiation to occur, and this stress will then lead intergranular corrosion to form a reasonably sharp microcrack instead of otherwise harmless pits, which may be the case if there was no applied stress.

The true SCC process, in that both external stress and corrosion are required simultaneously, is that of transgranular pseudo-cleavage, identified by the distinctive 
fluting pattern left behind on fracture surfaces. The propensity towards a pseudocleavage mechanism decreased as iodine concentrations increased. This is the same observation as originally reported by Serres et al [11], in exposures to iodisedmethanol but using different methods of characterisation. It was observed that increasing the iodine concentration, increased the critical stress-intensity factor required for an intergranular crack to begin cracking by pseudo-cleavage, which was suggested to be evidence that intergranular cracking was more susceptible to iodine concentration than cleavage cracking.

Intergranular iodine attack will occur preferentially at a grain boundary adjacent to the crack tip, where stress is highest. This will occur for both low and high iodine concentrations. However, as iodine concentrations are raised, there will be more free iodine to attack in the close proximity of the crack tip (perhaps even forming new microcracks). This will result in a blunter crack, and a lower stress intensity. The blunter crack will propagate deeper into the material than the sharper crack before becoming transgranular - thus larger iodine concentrations result in more intergranular cracking and equally, lower iodine concentrations result in increased amounts of transgranular cracking. It should be noted that these differences do not significantly affect the time to failure, suggesting that incubation time may be dominant in this case.

Increasing the iodine concentration was also observed to increase the proportion of ductile tearing observed on the fracture surfaces. When stress was varied, the TG region was observed to change location due to the decreased amount of IG cracking, but its measured area was not observed to change. However, as iodine concentration is increased, the proportion of the TG region decreased and the IG and ductile tearing areas increased. It seems that increased iodine concentrations have raised the stress intensity as the crack has progressed transgranularly through pseudo-cleavage and fluting. It may be that the presence of a higher concentration of iodine generated increased amount of cleavage, but further work is required to investigate this matter.

\subsection{Effect of Microstructure}

Macroscopic differences in crack direction between recrystallised and cold worked material have previously been observed, and have been used to justify particular mechanistic theories of PCI crack development $[5,35]$. From the main and incipient crack morphologies, it appears as though both RXA and CW cracks begin in a radial direction, but then after some depth the CW material begins to proceed at a particular angle $555^{\circ}$ 
from the radial direction, whereas the RXA cracks generally continue to propagate in the radial direction.

Therefore, the difference in cracking behaviour is most likely associated with a cracking mechanism other than intergranular pitting (i.e. fluting, cleavage, or ductile tearing). Crack initiation appears similar for both material conditions. Non-radial cracking in cold-worked material has previously been attributed to ductile tearing based upon early fractographic observations [36], however the current results, show cleavage and fluting to be dominant mechanisms in areas of the crack that progress non-radially. Indeed, the TG cracking through cleavage and fluting shown in Figure 5 for the coldworked material was taken from a region of non-radial crack propagation.

RXA cracks do radically change direction but only on the order of $\sim 10 \mu m$, which is likely due to local grain favourability. CW material appears to feature these granular changes in direction, but more macroscopically follows a more tangential than radial direction. A similar phenomenon has previously been observed in cold worked steel, with macroscopic crack directions generally taking the shortest and highest stressed route, but slightly deviating from the pathway towards the rolling direction [37].

Cleavage cracking has been observed to occur primarily on basal planes [1], and so the differences in texture between cold worked and recrystallized material could be thought to be partly responsible for the differences in cracking behaviour. As shown in the pole figures in Figure 1, there is a minor strengthening of the split basal texture after recrystallisation and a corresponding $30^{\circ}$ rotation of the prismatic planes. However, there is no major change in the basal plane orientation after recrystallisation, and the 30 - rotation of the prismatic planes is around the radial direction. It is difficult to see how these changes could be solely responsible for a crack deviating 55 towards the tangential direction.

There are several other potential explanations:

- The generally accepted explanation that was first suggested in 1974 [35], and mentioned again in 1998 [5], is based upon slip plane dislocation pileups and the observation of hydride platelets. If a leading dislocation is prevented from moving by a grain boundary or other barrier, dislocations on the same slip plane behind it may pile up. Zirconium atoms at the resultant dislocation pile ups are likely to be favourable sites for corrosion and in case the crack encounter them, the crack will propagate easier due to their reduced bonding to the matrix.

Hydride platelets are known to form at areas of high dislocation densities, and in cold worked material they are observed to form in lines perpendicular 
to the radial direction [38], indicating the presence of high dislocation densities in that orientation. It was therefore thought that cold worked material cracks in a non-radial direction due to the presence of dislocation pileups in non-radial directions $[5,35]$.

Research since 1974 have raised concerns about this explanation [39]. Most importantly that hydrides perpendicular to the radial direction are observed in recrystallised material (but not the accompanying tangential cracks), and that hydrides prefer to form along the basal plane of zirconium as opposed to forming purely based on local stress conditions [39]. Regardless, the increased tangential dislocation densities in CW material and their responsibility for steering the crack must be demonstrated in a better manner than by simply examining hydride platelets - the explanation is insufficient if not entirely incorrect.

- Due to the significant texture strengthening after recrystallisation, the basal planes are more strongly aligned in RXA material. This could lead to either fluting or cleavage being more favourable in one of the material conditions and should one of these mechanisms lead the crack in a non-radial direction, the difference in crack paths may be explained. This would however mean both conditions cracking at an angle, just to different degrees something that is not observed. Testing of intermediate textures and grain morphologies between the two examined in this work may provide more clarity on this.

- Cold worked material has high levels of residual stress. This could create a local stress state such that it is favourable for cracking to proceed at a non-radial angle. However, cracking observed in stress-relieved material subjected to I-SCC, were identical in nature to those of just cold-worked material indicating that residual stresses are not a major factor in defining the crack direction.

- Thermomechanical processing, particularly pilgering, of CW material leads to elongated grains with their major axis between radial and tangential directions, which could encourage non-radial cracking in two ways:

1. If an intergranular crack followed these grains it would propagate in a direction between radial and tangential, as that is the major axis on the long grains. Although this may account for some of the zig-zagging of granular scale that is observable within CW cracks, it does not account for their overall non-radial propagation at a larger scale, as 
fractography suggests transgranular cracking accounts for a large part of the crack.

2. Transgranular cracking will pass through the entirety of a grain, and if the subsequent neighbouring grain it encounters has an aligned crystallographic plane, the stress will induce transgranular cracking in that grain and in that direction. If that plane occurs radially in $\mathrm{CW}$ material, the crack will only progress $\sim 1 \mu \mathrm{m}$ before reaching the end of the grain. If instead the plane occurs in a more tangential direction, where CW grains are elongated the crack will progress up to $10 \mu \mathrm{m}$ before reaching the end of the grain. The overall crack would then be skewed in a direction parallel to the long axis of the elongated CW grains - in this case approximately $55^{\circ}$ from the radial direction.

Looking at the cold-worked incipient crack in Figure 4, it appears as though the first $50 \mu m$ progresses radially, with the crack then changing direction 50 - towards the tangential direction. With what we understand from the fractography that cracking begins with IG, before then proceeding through $T G$, it seems likely that this change occurs once $T G$ cracking begins. That IG and TG progress in different directions is further evidenced by the in-plane cracking observed in Figure 10. The second explanation thus seems the most likely based upon this work.

Optical maps of crack tips of the two material conditions show different crack morphologies. The split cracks within cold-worked material appear to travel in either the long direction of the grain, or the short directions, with the majority of the crack length occurring along the long direction. The small section of cracking that occurred in the short direction appears to be disconnected, although it is likely that they are connected in a plane not observed by a singular 2D map.

\section{Conclusions}

PCI has been investigated by inducing I-SCC in cold worked and recrystallised ZIRL0 cladding material through compressing C-ring specimens submerged in iodised ethanol at room temperature. Times to failure were comparable with experimental results available in literature despite differences in composition, geometry, stress state and most encouragingly, the iodine form. The effects of variations in stress and iodine concentration have been examined, as well as the difference between cracks within coldworked and recrystallised materials. For the first time for these materials, proportions of intergranular and transgranular cracking have been quantitatively investigated using 
SEM fractography, in relation to the metallurgical state, local microstructure and test parameters. The investigations of the crack propagation mechanisms revealed that:

- Higher macroscopic stress produced TG cracking, resulting in more direct and shorter crack lengths, thus reducing the time to failure.

- Larger areas of IG cracking occur with increasing iodine concentrations, although the overall time to failure was found to decrease, this is potentially related to the blunting of the cracks.

- Cracking of the recrystallised material proceeded radially, while the long and thin grains of the cold-worked material provided and assured a cracking direction that was skewed $\sim 50^{\circ}$ towards the tangential direction, progressing via the long axes of the grains.

This detailed examination of the cracking mechanism for I-SCC occurrence within ZIRLO cladding material at microstructural level, provides evidence that can be used for the development of a physically based model of PCI failure-.

\section{Acknowledgements}

This work was funded by the EPSRC - PACIFIC [EP/L018616/1] Programme and is supported by EPSRC Centre for Doctoral Training in Nuclear Fission- Next Generation Nuclear [EP/L015390/1]. It has been carried out as part of the PACE consortium on Pellet Cladding Interaction. The authors would like to thank Westinghouse Electric Sweden for providing the cladding materials used in this study.

\section{Data Avai lability}

The raw and processed data required to reproduce these findings is available upon request.

\section{References}

[1] B. Cox, Pellet-clad interaction (PCI) failures of zirconium alloy fuel cladding-a review, J. Nucl. Mater. 172 (1990) 249-292.

[2] Pellet-clad Interaction in Water Reactor Fuels, OECD, Aix-en-Provence, France, 2005.

[3] J.-S. Cheon, Y.-H. Koo, B.-H. Lee, J.-Y. Oh, D. -S. Sohn, Modelling of a Pellet-Clad Mechanical Interaction in LWR Fuel by Considering Gaseous Swelling, in: Pellet-Clad Interact. Water React. Fuels, 2004.

[4] S. Béguin, PCI-related Constraints on EDF PWRs and Associated Challenges, in: PelletClad Interact. Water React. Fuels, Organisation for Economic Co-Operation and Development - Nuclear Energy Agency, Paris, France, 2005: p. 53.

[5] P.. Sidky, Iodine stress corrosion cracking of Zircaloy reactor cladding: iodine 
chemistry (a review), J. Nucl. Mater. 256 (1998) 1-17.

[6] M. Peehs, H. Stehle, E. Steinberg, Out-of-Pile Testing of Iodine Stress Corrosion Cracking In Zircaloy Tubing in Relation to the Pellet Cladding Interaction Phenomenon, in: Zircon. Nucl. Ind. Fourth Int. Symp., Stratford-upon-Avon, 1979: pp. 244-260.

[7] A. Garlick, P.D. Wolfenden, Fracture of zirconium alloys in iodine vapour, J. Nucl. Mater. 41 (1971) 274-292.

[8] S. Beguin, PCI-related constraints on EDF PWRs and associated challenges, in: PelletClad Interact. Water React. Fuels, Organisation for Economic Co-Operation and Development - Nuclear Energy Agency, 75 - Paris (France); 548 p; ISBN 92-64-01157-9; Worldcat; Jul 2005; p. 53-62; Seminar: Pellet-clad Interaction in Water Reactor Fuels; Aix-en-Provence (France); 9-11 Mar 2004;, 2005: pp. 53-62.

[9] K. Une, Deformation and Fracture Behavior of Zircaloy-2 Deformed at Constant Strain Rate in Iodine Environment, (I), J. Nucl. Sci. Technol. 16 (1979) 577-587.

[10] D. B. Knorr, R.M. Pelloux, L.F.P. Van Swam, Effects of material condition on the iodine SCC susceptibility of zircaloy-2 cladding, J. Nucl. Mater. 110 (1982) 230-245.

[11] A. Serres, L. Fournier, M. Frégonèse, Q. Auzoux, D. Leboulch, The effect of iodine content and specimen orientation on stress corrosion crack growth rate in Zircaloy4, Corros. Sci. 52 (2010) 2001-2009.

[12] R. L. Jones, F. L. Yaggee, R. A. Stoehr, D. Cubicciotti, Threshold conditions for iodineinduced stress corrosion cracking of unirradiated Zircaloy-4 tubing under internal pressurization, J. Nucl. Mater. 82 (1979) 26-38.

[13] P. Jacques, F. Lefebvre, C. Lemaignan, Deformation-corrosion interactions for $\mathrm{Zr}$ alloys during I-SCC crack initiation: Part I: Chemical contributions, J. Nucl. Mater. 264 (1999) 239-248.

[14] S. A. Nikulin, A. B. Rozhnov, Corrosion Cracking of Zirconium Cladding Tubes (A Review). I. Methods of Study and Mechanisms of Fracture, Met. Sci. Heat Treat. 47 (2005) 7179.

[15] S.B. Farina, G.S. Duffó, J.R. Galvele, Stress Corrosion Cracking of Zirconium and Zircaloy-4 in Iodine-Alcoholic Solutions, Corrosion. 59 (2003) 436-442.

[16] H. Ohara, Fuel behavior during power ramp tests, Proc. Int. Top. Mtg. LWR Fuel Performance, West Palm Beach, April 17-21, 1994. 674 (1991).

[17] C. Anghel, A.-M.A. Holston, G. Lysell, R. Jakobsson, S. Karlsson, J. Flygare, E. Sund, Mahmood, T. Sheikh, An Out-of-Pile Method to Investigate Iodine-induced SCC of Irradiated Cladding, Top Fuel 2009, Paris, Fr. Sept. 6-10, 2009. (2009) 823-834.

[18] A.V. Gomez Sanchez, S.B. Farina, G.S. Duffó, Effect of temperature on the stress corrosion cracking of Zircaloy-4 in iodine alcoholic solutions, Corros. Sci. 49 (2007) 3112-3117.

[19] V. Francon, M. Fregonese, H. Abe, Y. Watanabe, Iodine-Induced Stress Corrosion Cracking of Zircaloy-4: Identification of Critical Parameters Involved in Intergranular to Transgranular Crack Propagation, Solid State Phenom. 183 (2011) 4956.

[20] C. Gillen, Understanding the Mechanisms of Pellet Cladding Interaction in Zr Alloys and their Influence on the Degradation of Light Water Reactor Fuel Assemblies, University of Manchester, 2020.

[21] ASTM G38 - 01(2013) Standard Practice for Making and Using C-Ring Stress-Corrosion Test Specimens, (n. d.).

[22] Y. Prawoto, J.R.P. Djuansjah, W. B.W. Nik, E. Enemuoh, Critical view on the usage of C-ring specimen for stress corrosion crack (SCC) test on orthopedic implant: Experimental, numerical and analytical approaches, Mater. Sci. Eng. C. 32 (2012) 
1271-1279.

[23] S. Dymek, M. Dollar, TEM investigation of age-hardenable Al 2519 alloy subjected to stress corrosion cracking tests, Mater. Chem. Phys. 81 (2003) 286-288.

[24] N. Eliaz, A. Shachar, B. Tal, D. Eliezer, Characteristics of hydrogen embrittlement, stress corrosion cracking and tempered martensite embrittlement in high-strength steels, Eng. Fail. Anal. 9 (2002) 167-184.

[25] Y.L. Wu, F.H. Froes, A. Alvarez, C.G. Li, J. Liu, Microstructure and properties of a new super-high-strength Al-Zn-Mg-Cu alloy C912, Mater. Des. 18 (1997) 211-215.

[26] S. Cao, Mechanical properties of zirconium alloys and zirconium hydrides predicted from density functional perturbation theory, Dalt. Trans. 44 (2015) 18769.

[27] R. Sejnoha, J.C. Wood, Iodine-Induced Stress Corrosion Cracking of Fixed Deflection Stressed Slotted Rings of Zircaloy Fuel Cladding, Zircon. Nucl. Ind. Fourth Int. Symp. (1979) 261.

[28] J.C. Wood, Factors affecting stress corrosion cracking of Zircaloy in iodine vapour, J. Nucl. Mater. 45 (1972) 105-122.

[29] C. Gillen, A. Garner, A. Plowman, C.P. Race, T. Lowe, C. Jones, K. L. Moore, P. Frankel, Advanced 3D characterisation of iodine induced stress corrosion cracks in zirconium alloys, Mater. Charact. 141 (2018).

[30] K. Une, Threshold Values Characterizing Iodine-Induced SCC of Zircaloys., Spec. Meet. Pellet-Cladding Interact. Water React. (1980) 226.

[31] M.H.A. Piro, D. Sunderland, S. Livingstone, J. Sercombe, W. Revie, A. Quastel, K. Terrani, C. Judge, A Review of Pellet-Clad Interaction Behavior in Zirconium Alloy Fuel Cladding, in: Ref. Modul. Mater. Sci. Mater. Eng., Elsevier, 2017.

[32] L. Fournier, A. Serres, Q. Auzoux, D. Leboulch, G.S.S. Was, Proton irradiation effect on microstructure, strain localization and iodine-induced stress corrosion cracking in Zircaloy-4, J. Nucl. Mater. 384 (2009) 38-47.

[33] M. Fregonese, C. Olagnon, N. Godin, A. Hamel, T. Douillard, Strain-hardening influence on iodine induced stress corrosion cracking of Zircaloy-4, J. Nucl. Mater. 373 (2008) $59-70$.

[34] M.L. Rossi, C.D. Taylor, First-principles insights into the nature of zirconiumiodine interactions and the initiation of iodine-induced stress-corrosion cracking, J. Nucl. Mater. 458 (2015) 1-10.

[35] B. van der Schaaf, Fracture of Zircaloy-2 in an Environment Containing Iodine, in: Zircon. Nucl. Appl. ASTM STP 551, 1974: p. 479.

[36] T.A. Roberts, R.L. Jones, D. Cubicciotti, A.K. Miller, H.F. Wachob, E. Smith, F. L. Yaggee, A Stress Corrosion Cracking Model for Pellet-Cladding Interaction Failures in Light-Water Reactor Fuel Rods, Zircon. Nucl. Ind. (Fourth Conf. Am. Soc. Test. Mater. (1979) 285-305.

[37] K. Arioka, T. Yamada, T. Terachi, G. Chiba, Influence of Carbide Precipitation and Rolling Direction on Intergranular Stress Corrosion Cracking of Austenitic Stainless Steels in Hydrogenated High-Temperature Water, Corrosion. 62 (2006) 568-575.

[38] M.R. Louthan, R.P. Marshall, Control of hydride orientation in zircaloy, J. Nucl. Mater. 9 (1963) 170-184.

[39] K. Manikrishna, A. Sain, I. Samajdar, G. Dey, D. Srivastava, S. Neogy, R. Tewari, S. Banerjee, Resistance to hydride formation in zirconium: An emerging possibility, Acta Mater. 54 (2006) 4665-4675. 


\section{InVEStigating IOdine-InduCEd Stress CORROSion CRACKing OF Zirconium AlLoYs using Quantitative Fractography}

Conor Gillen ${ }^{1 *}$, Alistair Garner ${ }^{1}$, Clara Anghel ${ }^{2}$ Philipp Frankel ${ }^{1}$

${ }^{1}$ Materials Performance Centre, School of Materials, The University of Manchester, Manchester M13 9PL, UK

${ }^{2}$ Westinghouse Electric Sweden AB, SE-721 63, Västerås, Sweden

*Corresponding Author

\section{Abstract}

Pellet Cladding Interaction (PCI) is a fuel failure phenomenon that limits power manoeuvrability of light water nuclear reactors with Iodine-Stress Corrosion Cracking (I-SCC) the most commonly hypothesised cause. To increase mechanistic understanding, ISCC tests have been performed by compressing zirconium alloy c-rings at fixed load while submerged in iodised ethanol. Samples left until failure produced fracture surfaces subsequently analysed using quantitative Scanning Electron Microscopy (SEM) fractography, and samples with cracks arrested part way produced incipient cracks assessed by optical imaging. The influence of microstructure, stress and iodine concentration on I-SCC performances has been assessed by comparison between cold-worked and recrystallised material.

Increasing the stress resulted in faster time to failure and produced smaller regions of intergranular cracking; explained using the stress intensity factor and its control over the prevalent mechanism, further evidenced by tests that removed either iodine or stress part way through cracking. Increasing iodine concentrations resulted in faster times to failure and more intergranular cracking indicating the importance of an incubation period and a possible crack blunting effect.

Cracks in cold-worked material progress at a non-radial angle, whereas cracks within recrystallised and stress-relieved material cracks progress radially. Analysis suggests that the mechanisms that lead to this difference may be related to grain morphology rather than residual stress as is typically suggested in the literature, with fractography and incipient cracks used as evidence. 


\section{Introduction}

PCI is a fuel failure phenomenon that Limits the power manoeuvrability of Light Water Reactors. Resistance to PCI type of degradation is an increasingly desirable attribute as nuclear power seeks to become a base load power source that can respond to power fluctuations in renewable energy sources.

As power is increased, differential thermal expansion of the fuel and cladding leads to regions of high tensile stress on the inner surface of the cladding. The effect is compounded by the production of fission gases during operation, leading to internal pressure increases and fuel swelling [1-3]. These aggressive fission products can attack the inner surface of the cladding and in combination with the stress can lead to fuel failures at stresses that would otherwise be within safe limits [4]. One of the fission products released during operation that can generate PCI degradation is iodine although the contributing effect of other fission products has not been ruled out $[1,5,6]$.

Mitigation against PCI failures in PWRs is achieved primarily by following strict operating limits on power ramp rates, ensuring that the stress encountered by the fuel cladding while fission products are present remains below an empirically derived threshold $[2,5,7]$. The risk of fuel failure through PCI is increased after Extended Reduced Power Operation (ERPO) and in high burnup fuel assemblies [8]. With the drive towards higher burnup and increased power flexibility in modern reactor designs, a better understanding of the PCI phenomenon is required in order to reduce or remove over-conservative empirically-derived stress thresholds whilst ensuring the integrity of the cladding.

The effect of stress $[7,9]$, metallurgical state [10-13] and iodine concentration $[1,6,14]$ upon I-SCC failure rates have been investigated previously, using mainly Zircaloy-2 or Zircaloy-4 material. However, little work has been done investigating the effect of external variables upon the cracking mechanism [11], a gap this work seeks to address and with the aforementioned increased drive for better PCI performance in Pressurised Water Reactors (PWRs), particularly more understanding is required for $\mathrm{Nb}$ containing alloys such as $\mathrm{ZIRLO}^{\mathrm{TM}}, 1$.

Previous attempts to simulate iodine-induced stress corrosion cracks in the laboratory have been performed using gaseous iodine at reactor temperature, as well as iodised alcohol solutions at room temperature for experimental practicality $[1,13,14]$. Iodised alcohol produces cracking with fractography revealing both intergranular and transgranular mechanisms, including both pseudo-cleavage and fluting as observed in reactor created cracks [15]. In addition, the propensity of cracking in both vapour 
and solution responds in the same way to changes in applied stress and iodine concentration [13-15]. The generally agreed upon crack mechanism in I-SCC failures in both experiments involves a crack initiating step through intergranular (IG) pitting, followed by a region of transgranular (TG) pseudo-cleavage cracking and finally ductile failure. In reactor pitting is often not observed $[16,17]$. A key observation is that fracture surfaces created in gaseous iodine show regions of entirely transgranular cracking, while those cracked in alcoholic solutions exhibit transgranular and intergranular cracking occurring simultaneously [15]. Key differences between using iodine in the two forms are: the sizes of the active species, with steric hinderance limiting the movement of iodised alcoholic compounds when compared with iodine vapour [18], and the large difference in temperature: room temperature versus reactor operating temperatures.

In this study, C-ring specimens prepared from cold-worked, stress-relieved and recrystallised ZIRLO cladding specimens, are loaded in compression at different levels and combinations of stress and iodised-ethanol concentrations in order to induce stress corrosion cracks. The PCI behaviour of this alloy has recently become more important with the increased desirability of power manoeuvrability in PWRs, and the laboratory tests described allow a wide range of parameters to be investigated far more easily than would be possible with irradiated samples. Failed samples are analysed and compared through fractographic analysis using scanning electron microscopy (SEM) in order to determine the effect of testing parameters on active cracking mechanism. By varying the testing parameters and analysing the effect upon the cracking mechanism, improved knowledge about the PCI cracking mechanism itself and how it can be controlled can be obtained [19]. Improved mechanistic understanding of I-SCC with detailed microstructural characterization will provide a better understanding of the PCI phenomenon that can help to improve PCI modelling.

1 - ZIRLO is a trademark of Westinghouse Electric Company LLC, its Affiliates and/or its Subsidiaries in the United States of America and may be registered in other countries throughout the world. All rights reserved. Unauthorized use is strictly prohibited. 


\section{Experimental Methods}

\section{1 Materials}

The material used in this study is the commercial alloy ZIRLO (Table 1), provided by Westinghouse, in cold-worked and recrystallised condition. The material was provided in tube form and both conditions had undergone typical commercial processing routes including forging and pilgering with a final annealing step for the recrystallised alloy. The typical cold-worked and recrystallised microstructures are highlighted in Figure 1. Additionally, some of the cold-worked material was heated under vacuum at $300^{\circ} \mathrm{C}$ for five hours allowing the effect of residual stress to be examined.

Cold worked ZIRLO material features long and thin grains with approximate dimensions of $2 \times 10 \mu \mathrm{m}$, with their major axis parallel with the axial direction of the tube, although with $30^{\circ}$ rotation towards the tangential direction due to the pilgering process. In the radial direction the CW grains are $\sim 2 \mu \mathrm{m}$ thick [20]. Recrystallisation leads to a strengthening of the split basal, a $30^{\circ}$ rotation of the prismatic planes away from the radial direction towards the tangential direction, and the formation of $\sim 2 \mu \mathrm{m}$ equiaxed grains in all directions. In both cases, a split basal texture exists in the radial direction. 
Table 1: Composition in wt.\% of ZIRLO alloy used in this study

\begin{tabular}{ccccccc}
\hline Element & $\mathbf{C r}$ & $\mathbf{F e}$ & $\mathbf{N b}$ & $\mathbf{N i}$ & $\mathbf{S n}$ & $\mathbf{Z r}$ \\
\hline Content (wt.\%) & $<0.01$ & 0.09 & 0.87 & $<0.01$ & 0.92 & bal. \\
\hline \hline
\end{tabular}

(a)
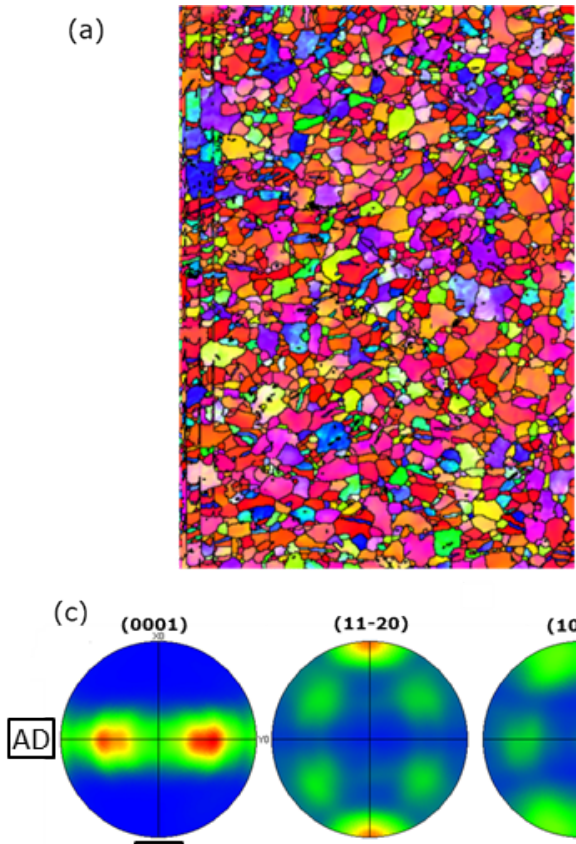

$\mathrm{TD}$

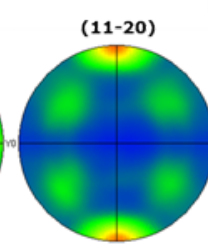

$(11-20)$
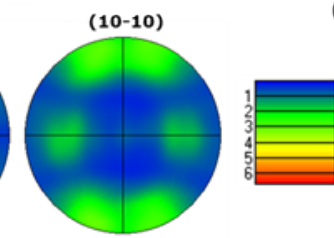

(b)
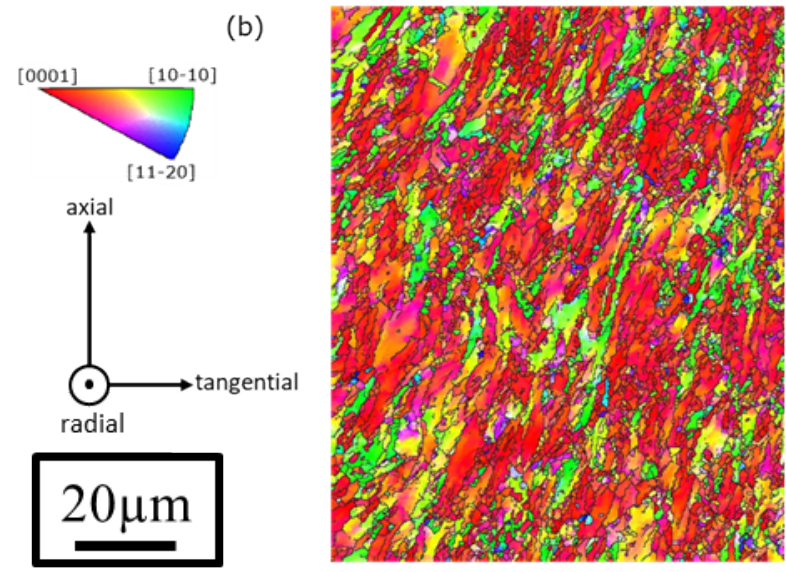

(d)

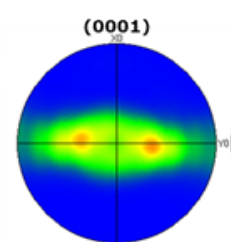

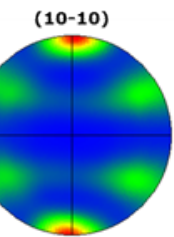

Figure 1 - Electron Backscatter Diffraction (EBSD) orientation maps in IPF-RD (radial tube direction) colouring for (a) Recrystallised and (b) cold-worked ZIRLO cladding tubes. Corresponding pole figures are shown for recrystallised and cold-worked conditions in (c) and (d) respectively.

The tubes were sectioned in order to produce C-ring samples for testing. The C-ring geometry is commonly used in stress corrosion cracking experiments, providing a region of stress concentration without notching so that crack initiation can be investigated [21-25]. The geometry of cladding tubes naturally lends itself to C-ring production and the maximum tensile stress when a displacement is exerted can be calculated using the following formula [21]:

Equation 1

$$
\sigma=\frac{3 E t\left(w-w_{0}\right)}{3 \pi R}
$$

where $E=$ elastic modulus (taken as $90 \mathrm{GPa}[26]$ ), $t=$ wall thickness, $w$ and $w_{0}$ are the slot width of the C-ring in the compressive direction during and before loading respectively, and $R$ is the radius. If the sample is stressed sufficiently as to undergo plastic deformation, this stress is multiplied by a plastic correction factor $f_{p}$, calculated by measuring the amount that the slot width changes when stressed by different displacements according to Sejnoha and Wood [27]: 
Equation 2

$$
f_{p}=\frac{w-\left(w_{u}+w_{R}\right)}{w-w_{0}}
$$

Where $w_{u}=$ the slot width on removing the load and $w_{R}$ the slot width arising from residual stress apparent when first cutting the ring. This factor was calculated for each of the stress levels used in this study. The manufactured samples had a surface area of $59 \mathrm{~cm}^{3}$ and a volume of $0.153 \mathrm{~cm}^{3}$

\subsection{Iodised Ethanol}

The experiments were conducted using $50 \mathrm{~mL}$ of an iodised ethanol solution of varying concentrations, from $0.1 \mathrm{~g}$ of iodine per litre of ethanol up to $20 \mathrm{~g}(\mathrm{I}) / \mathrm{L}(\mathrm{eth})$. This is equivalent to 0.1 to $20 \mathrm{mg}$ of iodine per $\mathrm{cm}^{3}$ of ethanol, units commonly used in previous studies of I-SCC. These values are also roughly equivalent to $10^{-4}$ to $2 \times 10^{-2}$ grams of iodine per $\mathrm{cm}^{2}$ of exposed zirconium. These concentrations were chosen to cover a similar range of iodine concentrations as Wood et al. [28], ranging from $1 \times 10^{-4}$ to $1 \times 10^{-2}$ $\mathrm{g} / \mathrm{cm}^{2}$. Samples tested only in ethanol, with no iodine present, did not exhibit failure. This was done as a controlled reference experiment to demonstrate iodine and not ethanol as the corrosive media.

\subsection{Tensile test conditions}

C-ring samples were tested in compression using an Instron 3344 tensile tester, partially submerged in iodised ethanol within a glass container as shown in Figure 2. Four different strategies were followed to investigate the various effects of iodine and applied stress. Additional tests also investigated the effects of metallurgical condition. All compressions took place at a rate of $2 \mathrm{~mm} / \mathrm{min}$ until the desired displacement (and thus stress) was reached at which point the sample was held under

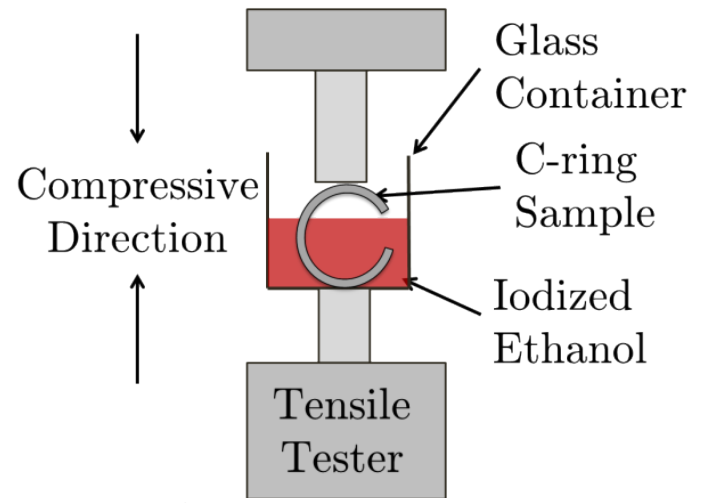

Figure 2 - Experimental setup for C-ring compression tests fixed load to negate plastic relaxation effects. Experiments ran until sample failure.

A displacement vs time graph of a typical test can be seen in Figure 3. Samples are loaded at constant displacement until reaching the desired displacement $(2 \mathrm{~mm})$ at which point they are held at fixed load until cracking begins. The tests were performed at 


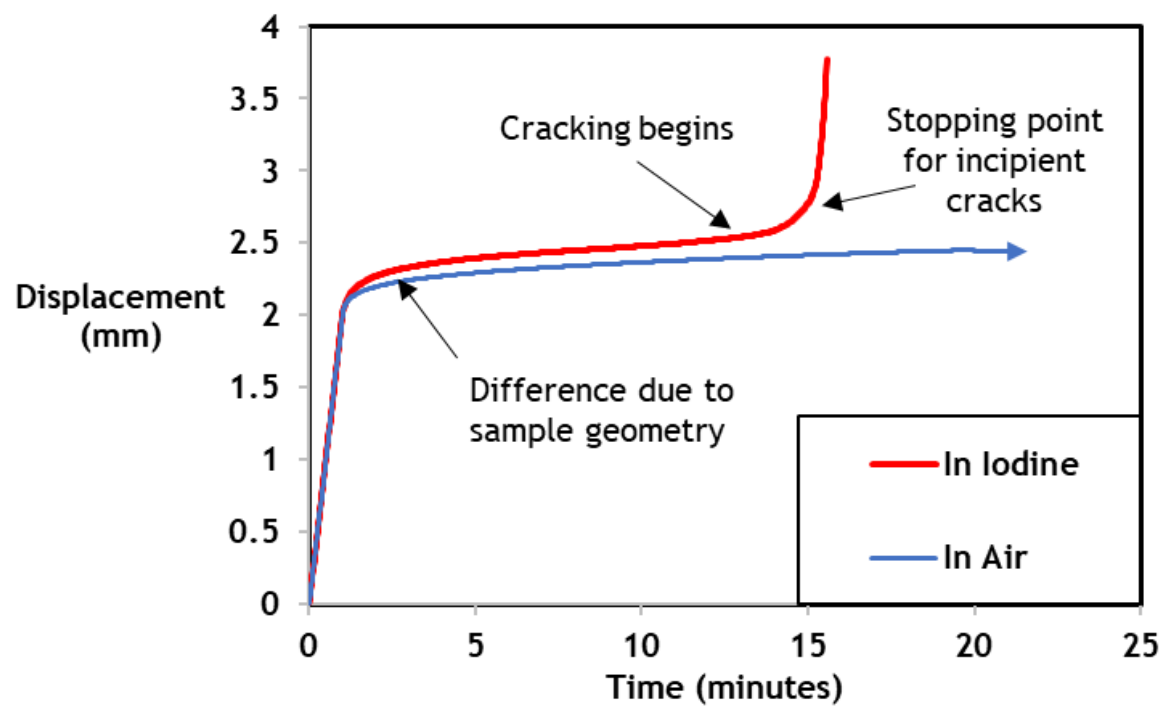

Figure 3 - Typical displacement vs time behaviour for cold-worked C-ring samples compressed to $2 \mathrm{~mm}$ and then held at fixed load in iodine-ethanol solution and in air.

properties differ between the material conditions, the resultant stresses on each material condition were different.

\subsubsection{Varying Macroscopic Stress}

The C-rings were loaded in a $1 \mathrm{mg} / \mathrm{cm}^{3}$ iodine ethanol solution until the desired maximum tensile stress ranging from 325 - $750 \mathrm{MPa}$ on the outer C-ring surface was reached (according to Equation 1), before holding at constant load.

Table 2: The four experiments investigating the effect of iodine concentration and applied stress.

\begin{tabular}{|c|c|c|c|c|}
\hline Experiment & $\begin{array}{c}\text { Initial crack } \\
\text { conditions }\end{array}$ & $\begin{array}{c}\text { Initial Crack } \\
\text { propagation stopped } \\
\text { at }\end{array}$ & $\begin{array}{c}\text { Second crack } \\
\text { growth } \\
\text { conditions }\end{array}$ & $\begin{array}{c}\text { Second crack } \\
\text { stopped at }\end{array}$ \\
\hline Varying Stress & $\begin{array}{c}397-794 \mathrm{MPa}, \\
1 \mathrm{mg} / \mathrm{cm}^{3}\end{array}$ & Ran until failure & $\mathrm{n} / \mathrm{a}$ & \\
\hline $\begin{array}{c}\text { Varying Iodine } \\
\text { Conc. }\end{array}$ & $\begin{array}{c}750 \mathrm{MPa}, \\
0.1-20 \mathrm{mg} / \mathrm{cm}^{3}\end{array}$ & Ran until failure & $\mathrm{n} / \mathrm{a}$ & \\
\hline $\begin{array}{c}\text { Temporary } \\
\text { removal of Stress }\end{array}$ & $750 \mathrm{MPa}$, & $\begin{array}{c}0.5 \mathrm{~mm} \text { change in } \\
\text { displacement }\end{array}$ & $750 \mathrm{MPa}$, & Ran until failure \\
\hline Removal of Iodine & $750 \mathrm{mPa} / \mathrm{cm}^{3}$ & \\
& $1 \mathrm{mg} / \mathrm{cm}^{3}$ & $\begin{array}{c}0.5-1 \mathrm{~mm} \text { change in } \\
\text { displacement }\end{array}$ & $750 \mathrm{MPa}$, in air & $\begin{array}{c}\text { Ran until failure } \\
\text { or crushed }\end{array}$ \\
\hline
\end{tabular}




\subsubsection{Varying lodine Concentration}

C-rings were compressed in varying concentrations of iodine ethanol solutions from $0.1-20 \mathrm{mg} / \mathrm{cm}^{3}$ at room temperature until a stress of $750 \mathrm{MPa}$ was reached, at which point constant load was maintained until sample failure or 15 hours elapsed (at which point the sample was considered to have not failed).

\subsubsection{Temporary Removal of Stress}

C-rings were compressed as before at fixed load upon reaching $750 \mathrm{MPa}$ all while in $1 \mathrm{mg} / \mathrm{cm}^{3}$ iodine ethanol solution. Cracking was observed to have begun by a change of $0.5 \mathrm{~mm}$ displacement occurring (as can be seen in Figure 3) at which point all applied stress was removed and the sample was left submerged in iodine for up to a week. Following this loading was recommenced in solution as before until sample failure.

\subsubsection{Removal of lodine}

Samples were compressed, until a maximum tensile load on the outer C-ring surface reached $750 \mathrm{MPa}$, at which point fixed load was applied, until a change of displacement ranging from $0.5-1 \mathrm{~mm}$ occurred indicative of incipient cracks. Stress was removed, and samples cleaned in ethanol. Samples were then subjected to the same compressive load as previously subjected to, but in air and not iodine solution. Either failure or survival was recorded following the second compression.

\subsubsection{Effect of Microstructure}

ZIRL0 C-ring samples of either cold-worked, stress-relieved or recrystallised material conditions were compressed to a range of stresses and iodine concentrations until sample failure.

\section{4 Microscopy}

Fractography was performed using a Zeiss EVO 60 SEM at $10 \mathrm{keV}$. Images were taken at 100 evenly spaced locations covering the fracture surface with the dominant fracture mechanism at each recorded, resulting in fracture surface maps of each sample. The proportions of the different fracture mechanisms could then be compared as applied stress and iodine concentrations are varied. This method generates quantitative data for comparison between samples, although there is an inherent subjectivity in each of the individual observations.

For comparison of incipient cracks, some tests were interrupted prematurely, and samples for cross sectional examination were prepared by grinding down non-failed 
samples from the axial direction of the cladding tube, followed by mechanical grinding and polishing, allowing optical images of the crack paths to be taken using a Zeiss Axio Scope. Areas of interest were selected as described in previously published work [29].

\section{Results}

\section{1 Incipient Cracks}

Typical arrested incipient cracks are shown in Figure 4, for both recrystallised and cold-worked material conditions. Cracking in recrystallised material progress with some directional changes on a scale similar to the grain size, but an overall radial direction is maintained. Coldworked material instead begins radially, before changing direction and progressing towards the tangential direction. Off-shoots progress from the main crack towards the opposite tangential direction.

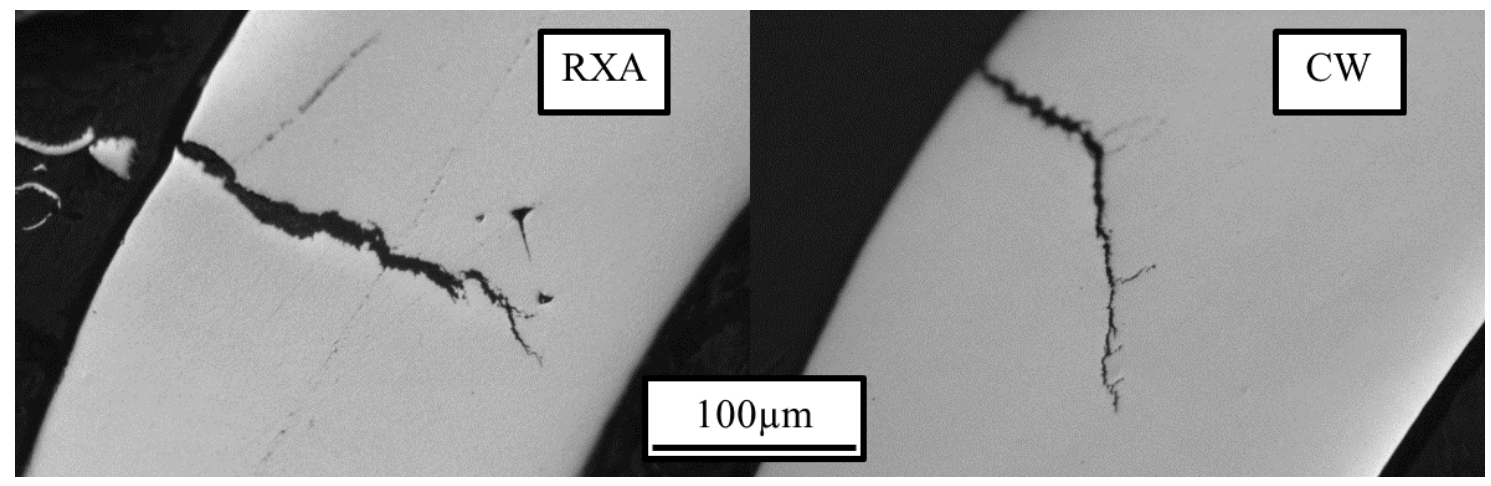

Figure 4 - Incipient cracks in RXA (left) and CW ( $r$ ight) ZIRLO material showing the difference in crack direction. Images are optical micrographs of crack tips polished down from each.

It is possible to use EBSD analysis to provide detailed microstructural analys is of the relationship of the crack tip to the microstructure [29], however that is limited to the very tip of the crack as correlating the crack to adjacent grains is much more difficult once the crack becomes open. In this work the crack path was monitored using fractography to provide information for many more grains.

\section{2 Effect of Microstructure}

The metallurgical state of the material was observed to have a large effect on the resulting crack path, as shown in the optical micrographs in Figure 4. Cracks in recrystallised ZIRLO material generally progress in a radial direction, taking the 
shortest route to the inner surface. In contrast, the cold worked material typically cracks in a direction approximately $50^{\circ}$ away from the radial direction following only a small amount of radial cracking, taking a considerably longer route from the outer to inner surface. In many cases, crack legs were observed to appear at 90 from the main crack path, and in the example in Figure 4, the crack leg was observed to develop into the main crack.

This radial vs non-radial cracking is also apparent in the TG images of RXA and CW material shown in Figure 5 (b) and (e) respectively. Features of similar size and shape to the grains shown in the EBSD map of Figure 1 are indicative of intergranular failure. In other places, RXA material exhibits flat transgranular cracking, with a river pattern typical of cleavage, whereas the CW TG image shows stepped cracking, with cleavage planes interrupted by perpendicular fluting steps. Regions identified as ductile tearing exhibit spherical microvoids, with areas of large ductility between them.

Tests were also performed on material that had been heated at $300^{\circ} \mathrm{C}$ in an attempt to reduce the residual stress and so examine its effect - these showed identical crack morphology to cold-worked material.

\subsection{Effect of Macroscopic Stress}

As the stress was increased, the time to failure for the ZIRLO samples submerged in $1 \mathrm{mg} / \mathrm{cm}^{3}$ iodised ethanol was observed to increase. Figure 6 compares results from the current investigation to results from the literature $[14,26,29]$. 


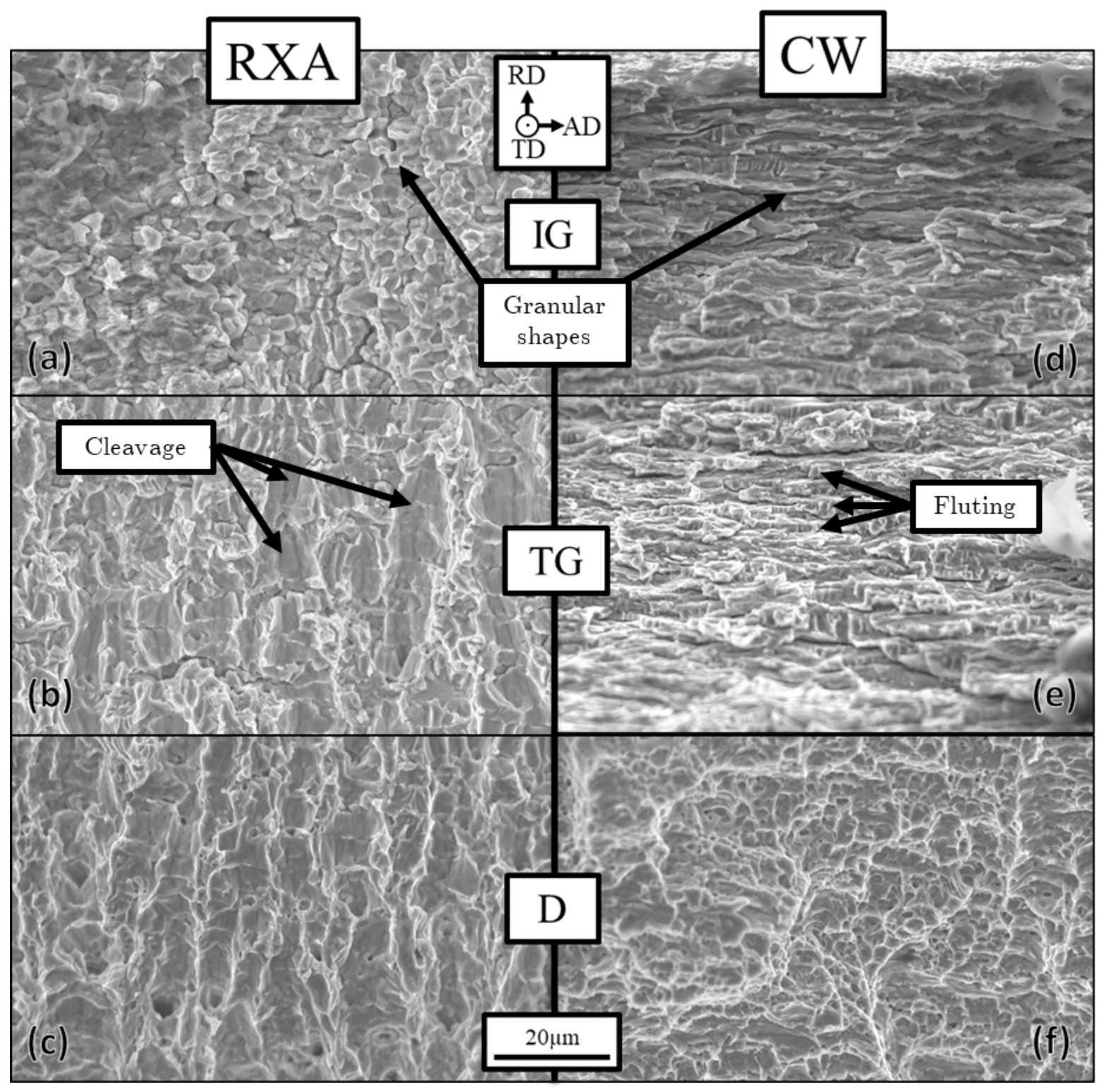

Figure 5 - Fracture surfaces from recrystallized (RX) $(a, b, c)$ and cold worked (CW) (d, e, f) ZIRLO Crings subjected to iodine stress corrosion cracking. Three areas of each are shown, showing the three typically observed regions dominated by the different fracture mechanisms; intergranular cracking (IG) $(a, d)$, transgranular cracking (TG) by pseudo-cleavage and fluting $(b, e)$ and ductile failure (D) (c, f). All images shown have the same magnification and orientation as specified. 


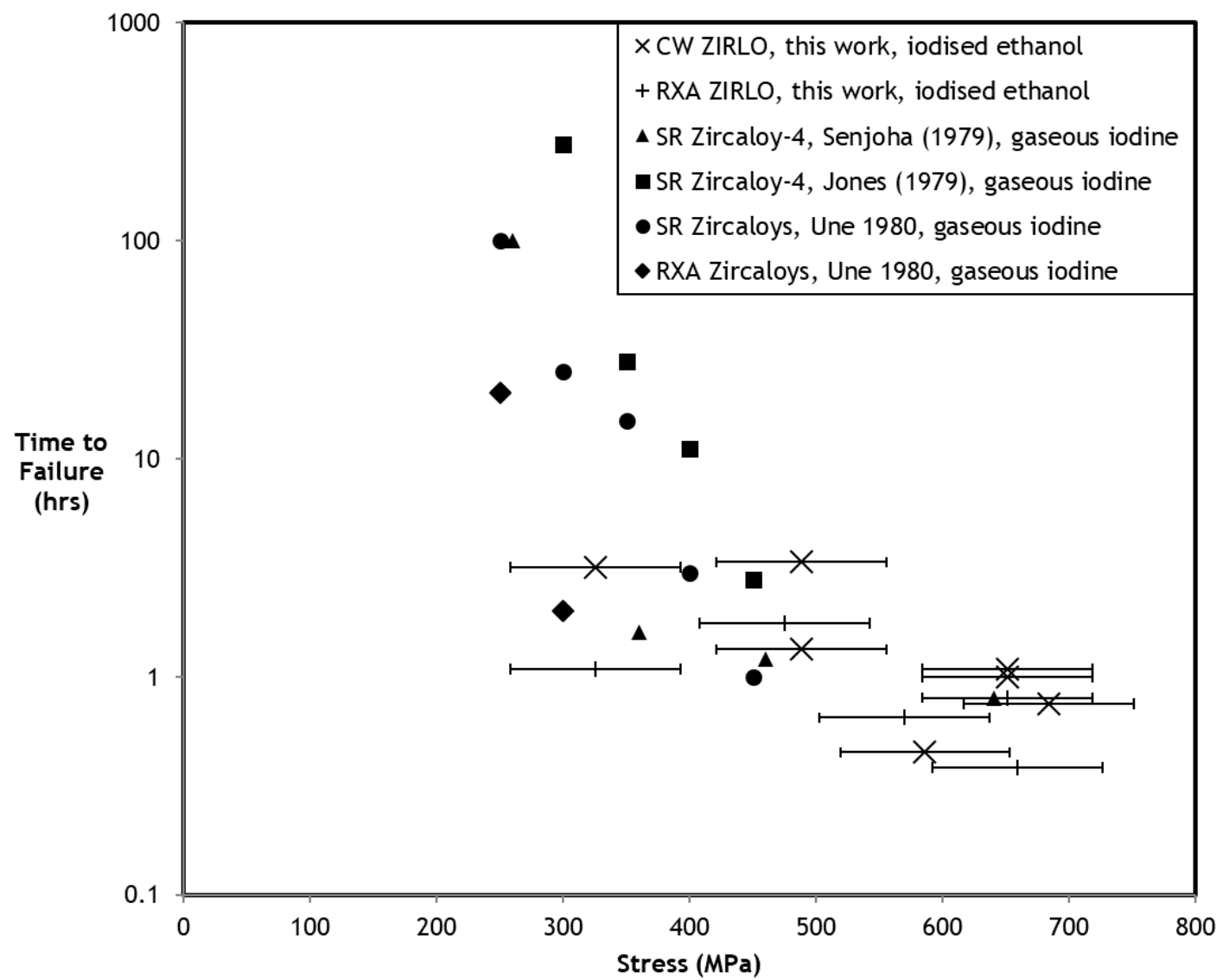

Figure 6 - Variation of time to failure versus changing stress for this work and selected others $[12,27,30]$

Corresponding changes in the proportions of IG, TG by pseudo-cleavage, and ductile cracking is evident on the fracture surface of ZIRLO samples submerged in $1 \mathrm{mg} / \mathrm{cm}^{3}$ iodised ethanol as shown in Figure 7, with increasing stress producing less intergranular cracking and more ductile failure, with the amount of transgranular cracking remaining fairly constant.

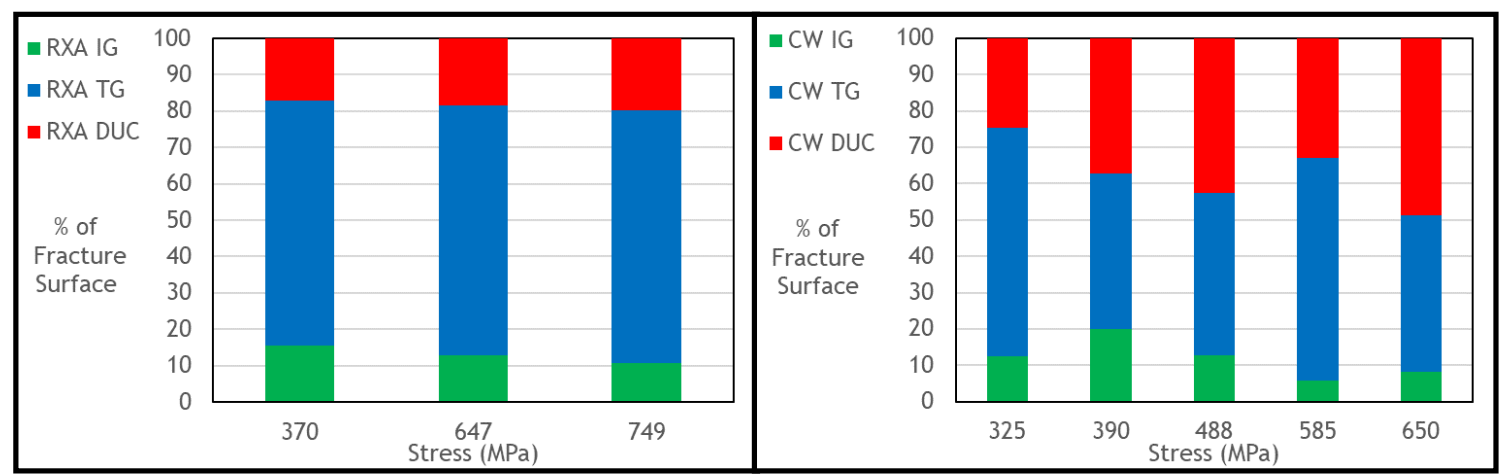

Figure 7 - The effects of applied stress and metallurgical state on the relative proportion of the fracture mechanisms observed by fractography for ZIRLO samples submerged in $1 \mathrm{mg} / \mathrm{cm}^{3}$ iodised ethanol at RT. 


\subsection{Effect of Iodine Concentration}

The effects of changing iodine concentration on the time to failure for C-rings compressed at a constant stress of $750 \mathrm{MPa}$ is shown in Figure 8 . As the iodine concentration was decreased, time to failures were observed to increase up until a concentration of approximately $1 \mathrm{mg} / \mathrm{cm}^{3}$. After this point, increases in concentration do not seem to affect times to failure. This trend is in agreement with previous results $[28,30]$. The value at which saturation begins $\left(1 \mathrm{mg} / \mathrm{cm}^{3}\right)$ is equivalent to $\sim 2 \times 10^{-3}$ $\mathrm{g}(\mathrm{I}) \mathrm{cm}^{-2}$ of zirconium cladding, of similar value to $6 \times 10^{-3} \mathrm{~g} / \mathrm{cm}^{2}$ observed by Wood [28]. The clear scatter in the data obfuscates any small difference that may exist in saturation concentration between the two material conditions, however they appear to be of the same magnitude.

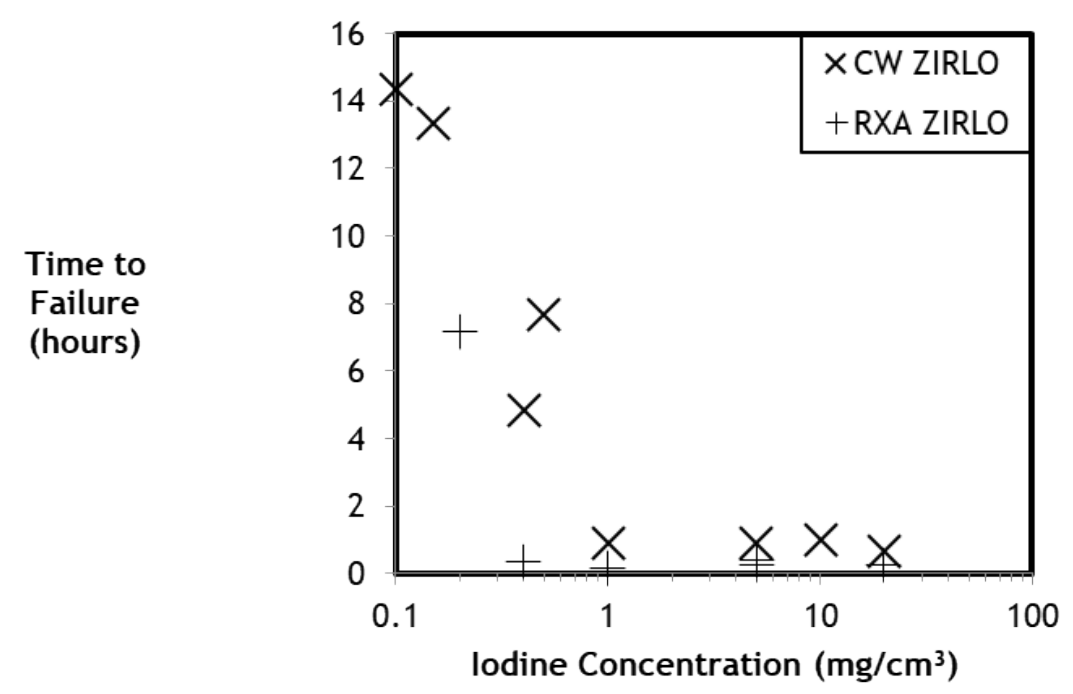

Figure 8 - The effect of changing iodine concentration on time to failure of cold-worked and recrystallised ZIRLO C-rings compressed and held at fixed $750 \mathrm{MPa}$ load until failure while submerged in iodised ethanol solution at RT.

The proportions of the resulting fracture surfaces dominated by each fracture mechanism are plotted in Figure 9. As the iodine concentration is increased, the amount of intergranular and ductile cracking is observed to increase, while the amount of transgranular cracking by pseudo-cleavage decreases. 


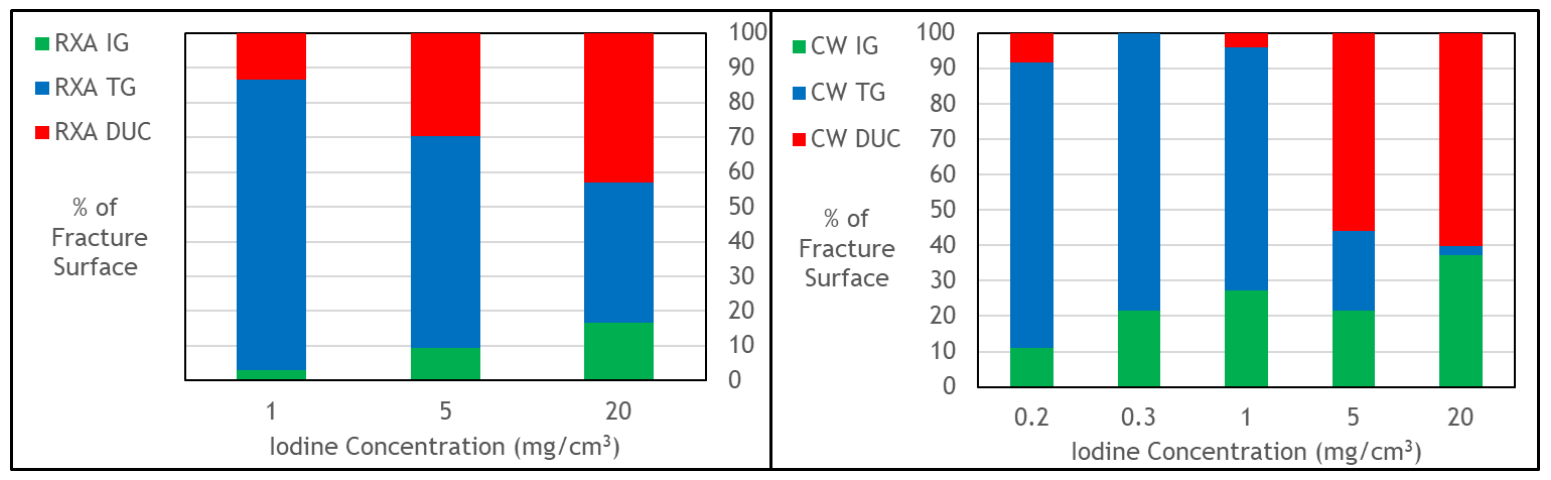

Figure 9 - The effects of changing iodine concentration and metallurgical state upon the distribution of different fracture mechanisms observed by fractography.

It should also be noted that for the exposure of ZIRLO samples to iodine concentrations less than $0.1 \mathrm{mg} / \mathrm{cm}^{3}$, cracking was not observed to occur within 15 hours, as for the ZIRL0 samples that were not exposed to iodine at all. Fracture surfaces from RXA samples tested at $<1 \mathrm{mg} / \mathrm{cm}^{3}$ were not fractographically examined.

\section{5 Temporary Removal of Stress}

Three ZIRL0 samples were partially cracked as described in Section 2.3.3, before being left submerged in iodised ethanol solution but under no applied stress for varying amounts of time, up to one week. The effect of corrosion could be observed by examining the maximum load that the samples reached before failure when subjected to the second compression test, as can be seen in Table 3.

Table 3: Effect of time spent submerged in iodised ethanol solution without stress upon failure load.

\begin{tabular}{|c|c|c|}
\hline Sample & \multicolumn{1}{c}{ Time submerged } & $\begin{array}{c}\text { \% Original } \\
\text { load reached }\end{array}$ \\
\hline (a) & 1 Hour & 97 \\
\hline (b) & 1 Day & 95 \\
\hline (c) & 1 Week & 79 \\
\hline
\end{tabular}

Fracture surfaces were then examined and characterised as illustrated in Figure 10. Sample (a), that was left submerged without stress for an hour, the shortest amount of time, shows no intergranular cracking in the middle of the sample. Sample (b), which was submerged for a day shows a small region of intergranular cracking in the middle of the sample, where only TG cracking would typically be expected. Sample (c), which was left submerged for one week, shows extensive intergranular cracking in the middle of the fracture surface. For samples (b) and (c), deep voids/cracks that extend into the material are surrounding the areas of intergranular cracking, which appear to be caused by intergranular corrosion. 

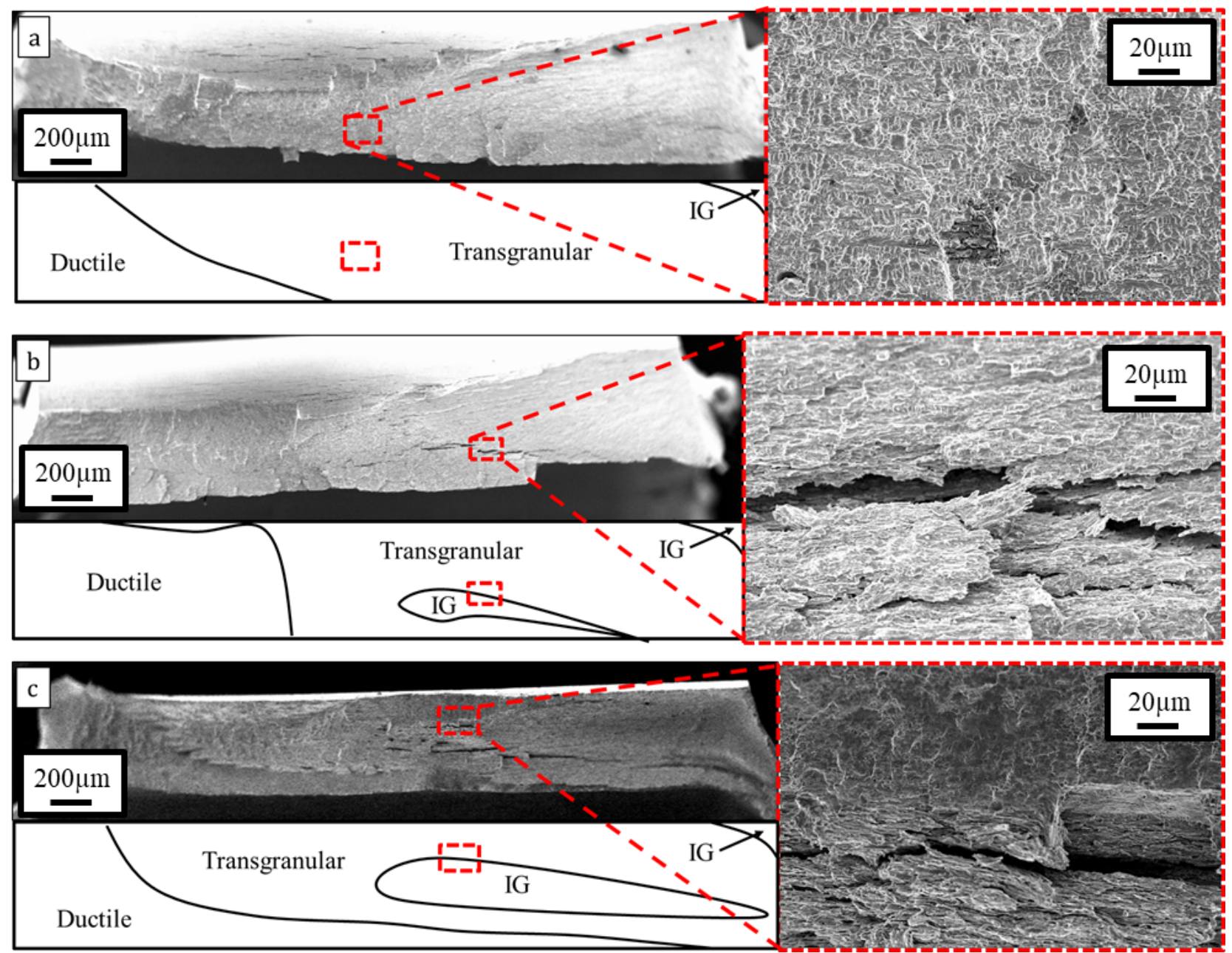

Figure 10 - Fractographic analysis of cold-worked ZIRLO samples partially cracked and then left submerged in iodised ethanol without an applied stress for a. One hour b. One day and c. One week, before being cracked until failure. The fractographic images with higher magnification (right side of the figure) show regions of IG cracking in the middle of the fracture surface or lack thereof.

\section{6 Removal of Iodine}

Three ZIRLO samples were partially cracked to different degrees as described in Section 2.3.4, before being submerged in ethanol and agitated for $\sim 30$ seconds, before being re-subjected to compression without the presence of iodine. When samples were held at fixed load, crack propagation could be seen by changes in crosshead displacement as in Figure 3. The amount of incipient cracking was controlled by removing the stress

Table 4: Effect of initial crack size (measured by change in displacement) upon whether the sample would fail when subsequently subjected to compression without iodine present.

\section{Sample $\quad \Delta$ displacement $(\mathrm{mm}) \quad$ Failure of sample on second compression?}

\begin{tabular}{|c|c|c|}
\hline $\mathbf{1}$ & 0.5 & $\times$ \\
\hline $\mathbf{2}$ & 0.75 & $\times$ \\
\hline $\mathbf{3}$ & 1 & $\checkmark$ \\
\hline
\end{tabular}


and iodine for different changes in this displacement. Whether samples failed or not when subjected to a second compression without iodine was then recorded, as can be seen in Table 4. Only the sample where the initial crack was allowed to grow the most was able to fail when subjected to subsequent compression without iodine present.

\section{Discussion}

Loading of C-rings in iodised ethanol solutions has allowed simulation of I-SCC in the laboratory whilst varying both stress and iodine concentration. The time to failure was observed to decrease as applied stress and iodine concentrations were increased. Fracture surfaces have been quantified by measuring the proportions of different cracking mechanisms present as iodine concentrations have been varied. Increasing stress has reduced the amount of intergranular cracking, whereas increasing iodine concentration has increased the amount of intergranular cracking. The metallurgical state of the material has not been observed to have a significant effect on the proportions of each cracking mechanism observed during fractography, however, incipient cracks in cold-worked and stress relieved ZIRLO have been observed to crack at a different angle than those in recrystallised material. The division of the cladding wall in three cracking patterns (Figure 5) and the time to failure show similarities (Figure 6 and Figure 8) when comparing the CW and RXA materials; this behaviour might be due to the similar split basal texture of all examined microstructures. For the current testing parameters, the time to failure for the CW and RXA materials is quite similar, which indicates that the rate limiting step is not affected by the microstructure of the material.

It is generally accepted within the literature that I-SCC proceeds through intergranular corrosion initially (although cracks beginning with transgranular cracking have been observed [31]), the crack propagates along susceptible grain boundaries (aided by stress) until the stress at the tip of this developing microcrack reaches a critical point. Transgranular cracking then occurs through pseudo-cleavage along basal planes $[12,28]$. This mechanism does not occur in an inert environment - a corrosive media is required and so is not expected to travel as fast as a true cleavage crack would as time for some chemical interaction at the crack tip is required for crack propagation to occur. It is therefore referred to as pseudo-cleavage; and is a SCC process. These cleavage planes can be connected when not aligned by prismatic plane failure, resulting in fluting, observable as the ' $Y$ ' shaped features in Figure 5 . The rest of the cladding wall may finally fail by ductile tearing due to microvoid 
coalescence [32]. This is not an SCC process, since it will occur in the complete absence of any corrosive media if the stress is high enough.

All fracture surfaces examined in this study agree with this sequence of progression of the different cracking mechanisms. Intergranular cracking occurred mostly at the outer C-ring edge where the crack was thought to initiate, and ductile tearing occurred on the inner surface, which would be last to fail. Transgranular cracking by pseudocleavage was mostly observed in the middle portion of the fracture surfaces, between the two other mechanisms, with the exception of the interrupted stress tests where prolonged exposure to iodine without an applied stress resulted in additional intergranular regions.

\section{1 The effect of Stress}

The effect of applied stress upon I-SCC failure rates is perhaps the most investigated relationship in literature and its general effect upon the cracking mechanism is well known and intuitive; increasing stress leads to a decrease in failure times, with the presented results in good agreement with trends reported in the literature $[12,27,30]$. This relationship however was not the primary focus of this work. Macroscopic stress has been previously shown to relate to the increased dominance of TG cracking at higher stresses, a mechanism much faster than IG cracking based upon acoustic emission data and previous comparison of fracture surfaces relative to failure times $[1,33]$. It is this relationship that the current work has sought to investigate. As can be seen from Figure 7, samples under higher stresses exhibit fracture surfaces with less intergranular cracking and more ductile tearing, a transgranular mechanism. For samples undergoing larger macroscopic stress the critical stress intensity factor at which ductile tearing can occur will be reached with a smaller crack length, and thus those samples under large stresses will undergo larger amounts of ductile tearing and will therefore have a decreased time to failure. This is because ductile tearing does not require a chemical contribution and is a fast crack propagation mechanism. It should be noted that the sharpness of the crack tip may also have an important contribution. With less time exposed to iodine, the crack that progresses under a higher macroscopic stress will be sharper, as less crack blunting corrosion can occur at the crack tip.

It is expected that as the stress intensity factor at a crack tip $K$, increases and reaches critical values, different mechanisms of I-SCC are enabled, as shown below:

Table 5: Dominant crack mechanisms for varying stress intensity factors, where $K_{T G}$ is defined as the minimum stress intensity at which transgranular cracking can occur, and $K_{\text {Ductile }}$ the minimum for ductile tearing.

$$
\mathrm{K}: \quad 0<\mathrm{K}_{\mathrm{TG}}<\mathrm{K}_{\text {Ductile }}
$$

\begin{tabular}{l|l|l|l} 
Mechanism: & Intergranular Attack & Pseudo-Cleavage & Ductile
\end{tabular}


Thus the presented results can be explained by considering that the stress intensity factor $K$ can be defined using the macroscopic stress the sample is held at $\sigma$, and some function of the crack length $a$ denoted $F(a)$, such that: $K=\sigma F(a)$. Therefore, if the macroscopic stress is increased, the critical values of $K$ are reached for a smaller crack size and in a shorter amount of time. The resulting fracture surfaces therefore show reduced proportions of IG cracking mechanisms for higher stresses, as the $K_{T G}$ is reached before intergranular attack has had a chance to occur. A number of additional control experiments that temporarily removed stress and completely removed iodine were carried out to separate the effects of different parameters on the resulting fracture surfaces, and lend further evidence for different parts of this explanation.

Firstly, stress was temporarily removed from the sample once an incipient crack had formed. Iodine was then free to attack the zirconium at the crack tip. Only intergranular corrosion occurred, and not a transgranular mechanism, indicating that in the absence of stress the degradation occurs via intergranular attack. This intergranular degraded area was surrounded by areas of transgranular and ductile cracking, presumed to have occurred upon resumption of loading. This fracture surface is atypical for laboratory I-SCC, and only occurred due to the exposure conditions the samples were subjected to. Repeated loading and unloading with the presence of iodine is currently avoided to prevent PCI [8], however may otherwise produce similar fractographic features indicative of the stop-start loading. The presented results are evidence that providing sufficient iodine ( $0.1 \mathrm{mg} / \mathrm{cm}^{3}$ in these experiments) is available intergranular attack can occur with no applied stress, whereas pseudo-cleavage requires enough stress to occur, as suggested in Table 5.

In a second control test, iodine was removed from testing following the initiation and propagation of incipient cracks to different depths. Subsequent loading without iodine resulted in sample failure for one sample within which the incipient had been allowed to grow the longest. This test provides evidence that ductile tearing can only occur for large incipient cracks and thus large $K$, again as suggested in Table 5; but when high enough $K$ ductile tearing occurs does not require additional iodine to be present.

\section{2 The effect of Iodine concentration}

When examining time to failure (Figure 8), the effect of iodine concentrations may be split into two regimes: 
1. Regime of proportionality in which increasing iodine concentration leads to faster sample failure

2. Saturation regime, during which increases in iodine concentration have no effect upon time to failure.

The two are both observed in the literature $[1,5,28]$. There is a third regime also, not investigated in this work, which occurs at very low iodine concentrations, when there is not enough concentration of corrosive species for I-SCC to initiate by pitting.

The rate limiting step during the regime of proportionality is thought to be the chemical interactions that must take place - zirconium iodides of increasing complexity are thought to form until reaching the volatile $\mathrm{ZrI}_{4}$ molecule, removal of which is believed to be the fundamental mechanism of crack propagation [34]. More local iodine will increase the number of attacked $\mathrm{Zr}$ atoms and increase the likelihood and corrosion rate of the more complex zirconium iodides formation, thus the negative relationship between iodine concentration and time to failure makes sense during the regime of proportionality.

The saturation regime can be explained in the same context, should there be an abundant amount of iodine locally, the amount of zirconium available becomes the restrictive quantity, and further increasing iodine concentrations has no effect, for the investigated stress levels.

Interestingly, the clear change observed between the regime of proportionality and saturation with regard to time to failures is not discernible upon examination of fracture surfaces as shown in Figure 9. Increasing iodine concentrations have resulted in increased levels of intergranular cracking, a difference that persists through the saturation regime, indicating that although the rate limiting step is not affected some other steps may be.

That the amount of intergranular corrosion increases with iodine concentration suggests that in contrast to ductile failure, intergranular corrosion is driven by a chemical process, with stress merely assisting it but not being required. It is intuitive for an increased number of available iodine atoms to cause an increased amount of corrosion through the formation of zirconium iodides. If a protective oxide exists, rupture of that oxide by stress is necessary for crack initiation to occur, and this stress will then lead intergranular corrosion to form a reasonably sharp microcrack instead of otherwise harmless pits, which may be the case if there was no applied stress.

The true SCC process, in that both external stress and corrosion are required simultaneously, is that of transgranular pseudo-cleavage, identified by the distinctive 
fluting pattern left behind on fracture surfaces. The propensity towards a pseudocleavage mechanism decreased as iodine concentrations increased. This is the same observation as originally reported by Serres et al [11], in exposures to iodisedmethanol but using different methods of characterisation. It was observed that increasing the iodine concentration, increased the critical stress-intensity factor required for an intergranular crack to begin cracking by pseudo-cleavage, which was suggested to be evidence that intergranular cracking was more susceptible to iodine concentration than cleavage cracking.

Intergranular iodine attack will occur preferentially at a grain boundary adjacent to the crack tip, where stress is highest. This will occur for both low and high iodine concentrations. However, as iodine concentrations are raised, there will be more free iodine to attack in the close proximity of the crack tip (perhaps even forming new microcracks). This will result in a blunter crack, and a lower stress intensity. The blunter crack will propagate deeper into the material than the sharper crack before becoming transgranular - thus larger iodine concentrations result in more intergranular cracking and equally, lower iodine concentrations result in increased amounts of transgranular cracking. It should be noted that these differences do not significantly affect the time to failure, suggesting that incubation time may be dominant in this case.

Increasing the iodine concentration was also observed to increase the proportion of ductile tearing observed on the fracture surfaces. When stress was varied, the TG region was observed to change location due to the decreased amount of IG cracking, but its measured area was not observed to change. However, as iodine concentration is increased, the proportion of the TG region decreased and the IG and ductile tearing areas increased. It seems that increased iodine concentrations have raised the stress intensity as the crack has progressed transgranularly through pseudo-cleavage and fluting. It may be that the presence of a higher concentration of iodine generated increased amount of cleavage, but further work is required to investigate this matter.

\subsection{Effect of Microstructure}

Macroscopic differences in crack direction between recrystallised and cold worked material have previously been observed, and have been used to justify particular mechanistic theories of PCI crack development $[5,35]$. From the main and incipient crack morphologies, it appears as though both RXA and CW cracks begin in a radial direction, but then after some depth the CW material begins to proceed at a particular angle $555^{\circ}$ 
from the radial direction, whereas the RXA cracks generally continue to propagate in the radial direction.

Therefore, the difference in cracking behaviour is most likely associated with a cracking mechanism other than intergranular pitting (i.e. fluting, cleavage, or ductile tearing). Crack initiation appears similar for both material conditions. Non-radial cracking in cold-worked material has previously been attributed to ductile tearing based upon early fractographic observations [36], however the current results, show cleavage and fluting to be dominant mechanisms in areas of the crack that progress non-radially. Indeed, the TG cracking through cleavage and fluting shown in Figure 5 for the coldworked material was taken from a region of non-radial crack propagation.

RXA cracks do radically change direction but only on the order of $\sim 10 \mu m$, which is likely due to local grain favourability. CW material appears to feature these granular changes in direction, but more macroscopically follows a more tangential than radial direction. A similar phenomenon has previously been observed in cold worked steel, with macroscopic crack directions generally taking the shortest and highest stressed route, but slightly deviating from the pathway towards the rolling direction [37].

Cleavage cracking has been observed to occur primarily on basal planes [1], and so the differences in texture between cold worked and recrystallized material could be thought to be partly responsible for the differences in cracking behaviour. As shown in the pole figures in Figure 1, there is a minor strengthening of the split basal texture after recrystallisation and a corresponding $30^{\circ}$ rotation of the prismatic planes. However, there is no major change in the basal plane orientation after recrystallisation, and the 30 - rotation of the prismatic planes is around the radial direction. It is difficult to see how these changes could be solely responsible for a crack deviating 55 towards the tangential direction.

There are several other potential explanations:

- The generally accepted explanation that was first suggested in 1974 [35], and mentioned again in 1998 [5], is based upon slip plane dislocation pileups and the observation of hydride platelets. If a leading dislocation is prevented from moving by a grain boundary or other barrier, dislocations on the same slip plane behind it may pile up. Zirconium atoms at the resultant dislocation pile ups are likely to be favourable sites for corrosion and in case the crack encounter them, the crack will propagate easier due to their reduced bonding to the matrix.

Hydride platelets are known to form at areas of high dislocation densities, and in cold worked material they are observed to form in lines perpendicular 
to the radial direction [38], indicating the presence of high dislocation densities in that orientation. It was therefore thought that cold worked material cracks in a non-radial direction due to the presence of dislocation pileups in non-radial directions $[5,35]$.

Research since 1974 have raised concerns about this explanation [39]. Most importantly that hydrides perpendicular to the radial direction are observed in recrystallised material (but not the accompanying tangential cracks), and that hydrides prefer to form along the basal plane of zirconium as opposed to forming purely based on local stress conditions [39]. Regardless, the increased tangential dislocation densities in CW material and their responsibility for steering the crack must be demonstrated in a better manner than by simply examining hydride platelets - the explanation is insufficient if not entirely incorrect.

- Due to the significant texture strengthening after recrystallisation, the basal planes are more strongly aligned in RXA material. This could lead to either fluting or cleavage being more favourable in one of the material conditions and should one of these mechanisms lead the crack in a non-radial direction, the difference in crack paths may be explained. This would however mean both conditions cracking at an angle, just to different degrees something that is not observed. Testing of intermediate textures and grain morphologies between the two examined in this work may provide more clarity on this.

- Cold worked material has high levels of residual stress. This could create a local stress state such that it is favourable for cracking to proceed at a non-radial angle. However, cracking observed in stress-relieved material subjected to I-SCC, were identical in nature to those of just cold-worked material indicating that residual stresses are not a major factor in defining the crack direction.

- Thermomechanical processing, particularly pilgering, of CW material leads to elongated grains with their major axis between radial and tangential directions, which could encourage non-radial cracking in two ways:

1. If an intergranular crack followed these grains it would propagate in a direction between radial and tangential, as that is the major axis on the long grains. Although this may account for some of the zig-zagging of granular scale that is observable within CW cracks, it does not account for their overall non-radial propagation at a larger scale, as 
fractography suggests transgranular cracking accounts for a large part of the crack.

2. Transgranular cracking will pass through the entirety of a grain, and if the subsequent neighbouring grain it encounters has an aligned crystallographic plane, the stress will induce transgranular cracking in that grain and in that direction. If that plane occurs radially in $\mathrm{CW}$ material, the crack will only progress $\sim 1 \mu \mathrm{m}$ before reaching the end of the grain. If instead the plane occurs in a more tangential direction, where CW grains are elongated the crack will progress up to $10 \mu \mathrm{m}$ before reaching the end of the grain. The overall crack would then be skewed in a direction parallel to the long axis of the elongated CW grains - in this case approximately $55^{\circ}$ from the radial direction.

Looking at the cold-worked incipient crack in Figure 4, it appears as though the first $50 \mu m$ progresses radially, with the crack then changing direction 50 - towards the tangential direction. With what we understand from the fractography that cracking begins with IG, before then proceeding through $T G$, it seems likely that this change occurs once $T G$ cracking begins. That IG and TG progress in different directions is further evidenced by the in-plane cracking observed in Figure 10. The second explanation thus seems the most likely based upon this work.

Optical maps of crack tips of the two material conditions show different crack morphologies. The split cracks within cold-worked material appear to travel in either the long direction of the grain, or the short directions, with the majority of the crack length occurring along the long direction. The small section of cracking that occurred in the short direction appears to be disconnected, although it is likely that they are connected in a plane not observed by a singular 2D map.

\section{Conclusions}

PCI has been investigated by inducing I-SCC in cold worked and recrystallised ZIRL0 cladding material through compressing C-ring specimens submerged in iodised ethanol at room temperature. Times to failure were comparable with experimental results available in literature despite differences in composition, geometry, stress state and most encouragingly, the iodine form. The effects of variations in stress and iodine concentration have been examined, as well as the difference between cracks within coldworked and recrystallised materials. For the first time for these materials, proportions of intergranular and transgranular cracking have been quantitatively investigated using 
SEM fractography, in relation to the metallurgical state, local microstructure and test parameters. The investigations of the crack propagation mechanisms revealed that:

- Higher macroscopic stress produced TG cracking, resulting in more direct and shorter crack lengths, thus reducing the time to failure.

- Larger areas of IG cracking occur with increasing iodine concentrations, although the overall time to failure was found to decrease, this is potentially related to the blunting of the cracks.

- Cracking of the recrystallised material proceeded radially, while the long and thin grains of the cold-worked material provided and assured a cracking direction that was skewed $\sim 50^{\circ}$ towards the tangential direction, progressing via the long axes of the grains.

This detailed examination of the cracking mechanism for I-SCC occurrence within ZIRLO cladding material at microstructural level, provides evidence that can be used for the development of a physically based model of PCI failure.

\section{Acknowledgements}

This work was funded by the EPSRC - PACIFIC [EP/L018616/1] Programme and is supported by EPSRC Centre for Doctoral Training in Nuclear Fission- Next Generation Nuclear [EP/L015390/1]. It has been carried out as part of the PACE consortium on Pellet Cladding Interaction. The authors would like to thank Westinghouse Electric Sweden for providing the cladding materials used in this study.

\section{Data Avai lability}

The raw and processed data required to reproduce these findings is available upon request.

\section{References}

[1] B. Cox, Pellet-clad interaction (PCI) failures of zirconium alloy fuel cladding-a review, J. Nucl. Mater. 172 (1990) 249-292.

[2] Pellet-clad Interaction in Water Reactor Fuels, OECD, Aix-en-Provence, France, 2005.

[3] J.-S. Cheon, Y.-H. Koo, B.-H. Lee, J.-Y. Oh, D. -S. Sohn, Modelling of a Pellet-Clad Mechanical Interaction in LWR Fuel by Considering Gaseous Swelling, in: Pellet-Clad Interact. Water React. Fuels, 2004.

[4] S. Béguin, PCI-related Constraints on EDF PWRs and Associated Challenges, in: PelletClad Interact. Water React. Fuels, Organisation for Economic Co-Operation and Development - Nuclear Energy Agency, Paris, France, 2005: p. 53.

[5] P.. Sidky, Iodine stress corrosion cracking of Zircaloy reactor cladding: iodine 
chemistry (a review), J. Nucl. Mater. 256 (1998) 1-17.

[6] M. Peehs, H. Stehle, E. Steinberg, Out-of-Pile Testing of Iodine Stress Corrosion Cracking In Zircaloy Tubing in Relation to the Pellet Cladding Interaction Phenomenon, in: Zircon. Nucl. Ind. Fourth Int. Symp., Stratford-upon-Avon, 1979: pp. 244-260.

[7] A. Garlick, P.D. Wolfenden, Fracture of zirconium alloys in iodine vapour, J. Nucl. Mater. 41 (1971) 274-292.

[8] S. Beguin, PCI-related constraints on EDF PWRs and associated challenges, in: PelletClad Interact. Water React. Fuels, Organisation for Economic Co-Operation and Development - Nuclear Energy Agency, 75 - Paris (France); 548 p; ISBN 92-64-01157-9; Worldcat; Jul 2005; p. 53-62; Seminar: Pellet-clad Interaction in Water Reactor Fuels; Aix-en-Provence (France); 9-11 Mar 2004;, 2005: pp. 53-62.

[9] K. Une, Deformation and Fracture Behavior of Zircaloy-2 Deformed at Constant Strain Rate in Iodine Environment, (I), J. Nucl. Sci. Technol. 16 (1979) 577-587.

[10] D. B. Knorr, R.M. Pelloux, L.F.P. Van Swam, Effects of material condition on the iodine SCC susceptibility of zircaloy-2 cladding, J. Nucl. Mater. 110 (1982) 230-245.

[11] A. Serres, L. Fournier, M. Frégonèse, Q. Auzoux, D. Leboulch, The effect of iodine content and specimen orientation on stress corrosion crack growth rate in Zircaloy4, Corros. Sci. 52 (2010) 2001-2009.

[12] R. L. Jones, F. L. Yaggee, R. A. Stoehr, D. Cubicciotti, Threshold conditions for iodineinduced stress corrosion cracking of unirradiated Zircaloy-4 tubing under internal pressurization, J. Nucl. Mater. 82 (1979) 26-38.

[13] P. Jacques, F. Lefebvre, C. Lemaignan, Deformation-corrosion interactions for $\mathrm{Zr}$ alloys during I-SCC crack initiation: Part I: Chemical contributions, J. Nucl. Mater. 264 (1999) 239-248.

[14] S. A. Nikulin, A. B. Rozhnov, Corrosion Cracking of Zirconium Cladding Tubes (A Review). I. Methods of Study and Mechanisms of Fracture, Met. Sci. Heat Treat. 47 (2005) 7179.

[15] S.B. Farina, G.S. Duffó, J.R. Galvele, Stress Corrosion Cracking of Zirconium and Zircaloy-4 in Iodine-Alcoholic Solutions, Corrosion. 59 (2003) 436-442.

[16] H. Ohara, Fuel behavior during power ramp tests, Proc. Int. Top. Mtg. LWR Fuel Performance, West Palm Beach, April 17-21, 1994. 674 (1991).

[17] C. Anghel, A.-M.A. Holston, G. Lysell, R. Jakobsson, S. Karlsson, J. Flygare, E. Sund, Mahmood, T. Sheikh, An Out-of-Pile Method to Investigate Iodine-induced SCC of Irradiated Cladding, Top Fuel 2009, Paris, Fr. Sept. 6-10, 2009. (2009) 823-834.

[18] A.V. Gomez Sanchez, S.B. Farina, G.S. Duffó, Effect of temperature on the stress corrosion cracking of Zircaloy-4 in iodine alcoholic solutions, Corros. Sci. 49 (2007) 3112-3117.

[19] V. Francon, M. Fregonese, H. Abe, Y. Watanabe, Iodine-Induced Stress Corrosion Cracking of Zircaloy-4: Identification of Critical Parameters Involved in Intergranular to Transgranular Crack Propagation, Solid State Phenom. 183 (2011) 4956.

[20] C. Gillen, Understanding the Mechanisms of Pellet Cladding Interaction in Zr Alloys and their Influence on the Degradation of Light Water Reactor Fuel Assemblies, University of Manchester, 2020.

[21] ASTM G38 - 01(2013) Standard Practice for Making and Using C-Ring Stress-Corrosion Test Specimens, (n. d.).

[22] Y. Prawoto, J.R.P. Djuansjah, W. B.W. Nik, E. Enemuoh, Critical view on the usage of C-ring specimen for stress corrosion crack (SCC) test on orthopedic implant: Experimental, numerical and analytical approaches, Mater. Sci. Eng. C. 32 (2012) 
1271-1279.

[23] S. Dymek, M. Dollar, TEM investigation of age-hardenable Al 2519 alloy subjected to stress corrosion cracking tests, Mater. Chem. Phys. 81 (2003) 286-288.

[24] N. Eliaz, A. Shachar, B. Tal, D. Eliezer, Characteristics of hydrogen embrittlement, stress corrosion cracking and tempered martensite embrittlement in high-strength steels, Eng. Fail. Anal. 9 (2002) 167-184.

[25] Y.L. Wu, F.H. Froes, A. Alvarez, C.G. Li, J. Liu, Microstructure and properties of a new super-high-strength Al-Zn-Mg-Cu alloy C912, Mater. Des. 18 (1997) 211-215.

[26] S. Cao, Mechanical properties of zirconium alloys and zirconium hydrides predicted from density functional perturbation theory, Dalt. Trans. 44 (2015) 18769.

[27] R. Sejnoha, J.C. Wood, Iodine-Induced Stress Corrosion Cracking of Fixed Deflection Stressed Slotted Rings of Zircaloy Fuel Cladding, Zircon. Nucl. Ind. Fourth Int. Symp. (1979) 261.

[28] J.C. Wood, Factors affecting stress corrosion cracking of Zircaloy in iodine vapour, J. Nucl. Mater. 45 (1972) 105-122.

[29] C. Gillen, A. Garner, A. Plowman, C.P. Race, T. Lowe, C. Jones, K. L. Moore, P. Frankel, Advanced 3D characterisation of iodine induced stress corrosion cracks in zirconium alloys, Mater. Charact. 141 (2018).

[30] K. Une, Threshold Values Characterizing Iodine-Induced SCC of Zircaloys., Spec. Meet. Pellet-Cladding Interact. Water React. (1980) 226.

[31] M.H.A. Piro, D. Sunderland, S. Livingstone, J. Sercombe, W. Revie, A. Quastel, K. Terrani, C. Judge, A Review of Pellet-Clad Interaction Behavior in Zirconium Alloy Fuel Cladding, in: Ref. Modul. Mater. Sci. Mater. Eng., Elsevier, 2017.

[32] L. Fournier, A. Serres, Q. Auzoux, D. Leboulch, G.S.S. Was, Proton irradiation effect on microstructure, strain localization and iodine-induced stress corrosion cracking in Zircaloy-4, J. Nucl. Mater. 384 (2009) 38-47.

[33] M. Fregonese, C. Olagnon, N. Godin, A. Hamel, T. Douillard, Strain-hardening influence on iodine induced stress corrosion cracking of Zircaloy-4, J. Nucl. Mater. 373 (2008) $59-70$.

[34] M.L. Rossi, C.D. Taylor, First-principles insights into the nature of zirconiumiodine interactions and the initiation of iodine-induced stress-corrosion cracking, J. Nucl. Mater. 458 (2015) 1-10.

[35] B. van der Schaaf, Fracture of Zircaloy-2 in an Environment Containing Iodine, in: Zircon. Nucl. Appl. ASTM STP 551, 1974: p. 479.

[36] T.A. Roberts, R.L. Jones, D. Cubicciotti, A.K. Miller, H.F. Wachob, E. Smith, F. L. Yaggee, A Stress Corrosion Cracking Model for Pellet-Cladding Interaction Failures in Light-Water Reactor Fuel Rods, Zircon. Nucl. Ind. (Fourth Conf. Am. Soc. Test. Mater. (1979) 285-305.

[37] K. Arioka, T. Yamada, T. Terachi, G. Chiba, Influence of Carbide Precipitation and Rolling Direction on Intergranular Stress Corrosion Cracking of Austenitic Stainless Steels in Hydrogenated High-Temperature Water, Corrosion. 62 (2006) 568-575.

[38] M.R. Louthan, R.P. Marshall, Control of hydride orientation in zircaloy, J. Nucl. Mater. 9 (1963) 170-184.

[39] K. Manikrishna, A. Sain, I. Samajdar, G. Dey, D. Srivastava, S. Neogy, R. Tewari, S. Banerjee, Resistance to hydride formation in zirconium: An emerging possibility, Acta Mater. 54 (2006) 4665-4675. 


\section{Declaration of interests}

૫ The authors declare that they have no known competing financial interests or personal relationships that could have appeared to influence the work reported in this paper.

$\square$ The authors declare the following financial interests/personal relationships which may be considered as potential competing interests:

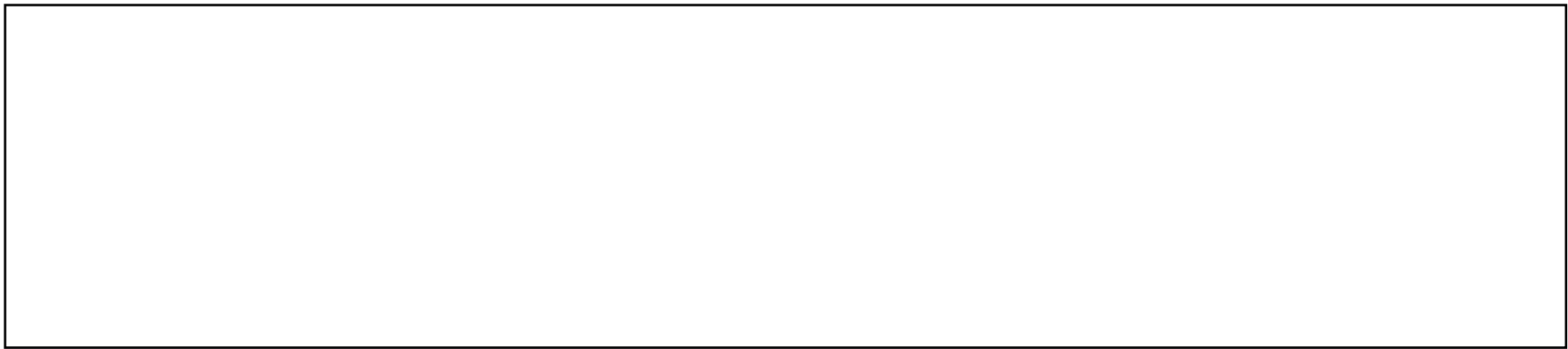


Conor Gillen

Conceptulisation, Methodology, Investigation, Writing - Original draft, reviewing and editing, Visualisation

Alistair Garner

Conceptulisation, Investigation, Writing - reviewing and editing

Clara Anghel

Writing - reviewing and editing

Philipp Frankel

Conceptulisation, Methodology, Resources, Writing - reviewing and editing, supervision, project administration, funding acquisition 